ESAIM: M2AN 48 (2014) 895-918

DOI: $10.1051 / \mathrm{m} 2 \mathrm{an} / 2013125$
ESAIM: Mathematical Modelling and Numerical Analysis

www.esaim-m2an.org

\title{
INTERFACE MODEL COUPLING VIA PRESCRIBED LOCAL FLUX BALANCE
}

\author{
Annalisa Ambroso ${ }^{1}$, Christophe Chalons $^{2}$, Frédéric Coquel $^{3}$ \\ AND ThOMAS GaliÉ ${ }^{1}$
}

\begin{abstract}
This paper deals with the non-conservative coupling of two one-dimensional barotropic Euler systems at an interface at $x=0$. The closure pressure laws differ in the domains $x<0$ and $x>0$, and a Dirac source term concentrated at $x=0$ models singular pressure losses. We propose two numerical methods. The first one relies on ghost state reconstructions at the interface while the second is based on a suitable relaxation framework. Both methods satisfy a well-balanced property for stationary solutions. In addition, the second method preserves mass conservation and exactly restores the prescribed singular pressure drops for both unsteady and steady solutions.
\end{abstract}

Mathematics Subject Classification. 35L50, 35L60, 35L65, 35L67, 35L81, 76M12.

Received February 15, 2012. Revised July 6, 2013

Published online April 24, 2014.

\section{INTRODUCTION}

Several problems from the Physics and Engineering yield nonlinear hyperbolic equations involving fluxfunctions and source terms which depend on the space variable. In the scalar setting, their mathematical analysis can trace back to the seminal work of Kruzhkov [38] assuming a smooth enough dependence for the non-homogeneous flux functions and source terms. As the consequence of the modeling of different natural phenomena, many situations of practical importance however require to handle closures exhibiting far less smooth non-homogeneities. The case of a flux-function which is discontinuous in the space variable has retained a considerable attention over the past two decades. Assuming an identically null source term (or say without real extra difficulties, a smooth enough non-homogeneous source term), its mathematical analysis has been initiated in the 1990s by Gimse, Risebro [21] and Diehl [18] in the scalar case, introducing the fruitful and mathematically deep topic of coupling two nonlinear hyperbolic equations in one space dimension at an interface at $x=0$. The proposed coupling framework is naturally conservative from the underlying Physics. A striking issue stays in the fact that there exists generically infinitely many possibilities to couple solutions in $x<0$ and $x>0$, which all satisfy the prescribed conservation condition. Bürger, Karlsen have then extensively analyzed this conservative

Keywords and phrases. Gas dynamics equations, interfacial coupling, measure valued load, relaxation method, coupled Riemann problem.

1 UPMC Univ Paris 06, UMR 7598, Laboratoire Jacques-Louis Lions, 75005 Paris, France.

2 Laboratoire de Mathématiques de Versailles, UMR 8100, Université de Versailles Saint-Quentin-en-Yvelines UFR des Sciences, bâtiment Fermat, 45 avenue des Etats-Unis, 78035 Versailles Cedex, France. christophe.chalons@uvsq.fr

3 CNRS \& Centre de Mathématiques Appliquées, U.M.R. 7641 Ecole Polytechnique, Route de Saclay, 91128 Palaiseau Cedex, France. frederic. coquel@cmap.polytechnique.fr 
coupling framework with respect to well-posedness (see [13] for a survey) and relevant numerical methods [14]. Several other contributions have been proposed and far from being exhaustive, we quote for instance $[1,6,7]$ and the references therein.

The case of source terms that are singular in the space variable has retained in comparison much less attention despite that many problems of importance actually involve source terms that are Dirac measures. We refer to the pioneering work by Diehl [17] in the setting of scalar equations. Let us also quote Gosse [27] for a well-balanced numerical strategy devoted to measure source terms. In the frame of the compressible fluid models, well-known situations correspond to the so-called singular pressure drops or head losses that are commonly encountered in various settings. They are responsible for a singular source term in the momentum equation (and/or the energy equation). A typical situation comes from free surface hydraulics where local losses result for instance from trash racks and slide gates. We refer the reader to the text-book by Hager [19] for a description together with several other examples where locally on purpose or not, the main flow either accelerates or is retarded. Another well-referenced situation corresponds to pressurized flows where singular pressure drops may arise from conduit bend, diffusors or mixing grids. There exists an enormous amount of data on loss semi-empirical laws which have been summarized, for example, by Idel'cik [34] or Miller [37]. In all the reported settings, the flux function does not depend on the space variable but by essence, the semi-empirical law at $x=0$ modeling the singular pressure drop resumes to a non-conservative coupling condition for the solutions in $x<0$ and $x>0$.

Recent applications actually rise the question of handling simultaneously a discontinuous flux-function at $x=0$ with a singular source term at the same space location. A major example comes from nuclear engineering but it may be encountered as well in the design of other large industrial setups. Their mathematical modeling often needs to treat the whole system as a collection of sub-systems where due to local operating conditions, distinct physical phenomena have to be accounted for. In addition, the transition from a given sub-system to the next one generally results in singular source terms. We refer to [8] within the frame of nuclear thermal hydraulics. As underlined in [8], existing ad hoc numerical methods to handle the reported non-conservative framework have revealed some shortcomings. This observation has assessed the need for mathematical investigations. The existence of semi-empirical laws (see $[8,36]$ ) that define the right trace of the coupled solution as a non-linear function of its left trace at $x=0$, referred to as transmission conditions hereafter, has motivated a series of studies devoted to well-posedness and to the derivation of efficient numerical methods. Transmission conditions are given a weak sense via a double IBVP formalism. The pioneering work [25] treats the coupling of scalar conservation laws and several extensions to the case of hyperbolic systems with possibly distinct size and involving general transmission conditions have been proposed for instance in [3,5]. Existence and uniqueness of a coupled solution to the double IBVP problem have been obtained in the frame of abstract systems in Lagrangian coordinates [4], where by nature nonlinear waves cannot interact with coupling interface. Existence of a solution can be established under fairly general assumptions for more general systems [11,15] but a difficulty lies in the fact that uniqueness may be lost. Loss of uniqueness is related to the resonance phenomena that may take place at the interface, when nonlinear waves associated with the fluid have locally vanishing propagation speed. Already in the scalar case [10], multiple solutions to the coupled Riemann problem can be exhibited when the coupling interface is resonant. The reported failure of uniqueness corresponds in fact to a general situation first described by Isaacson-Temple [29] in the scalar setting.

The present work falls within the frame of nuclear thermal hydraulics where the flows of interest are mostly if not always subsonic [8], namely the speed of propagation of the acoustic waves keeps values away from zero. As a consequence, the resonance phenomena is of artificial interest here and we thus restrict ourselves to subsonic coupled solutions for which existence and uniqueness is strongly expected to hold true. We propose an alternative approach to the double IBVP formalism for handling non-conservative coupling situations. We indeed suggest to set the coupled problem over the whole real line when observing that transmission conditions can as well understood to result from a Dirac measure source term concentrated at $x=0$ and which mass precisely defines the expected departure from the conservation property. The underlying Physics make this approach clearly natural. Motivated by singular pressure drops, we address the coupling of two Euler systems for barotropic 
gases, each equipped with a possibly distinct pressure laws, with a given Dirac measure at $x=0$ involved in the momentum equation. The property that the source term is a Dirac measure concentrated at $x=0$ makes the solutions of the coupled initial value problem for Riemann initial data to be self-similar. Subsonic self-similar solutions are made of two extreme non-linear waves separated by a standing wave accounting for the Dirac source term. Due to the non-linearities in the governing PDEs, the derivation of exact Riemann solutions is however cumbersome and we rely on convenient approximations of the latter. A sharp resolution of the singular pressure drops is the main goal of the present paper. We propose two methods with distinctive properties regarding the accuracy with which the pressure drops are restored. Both are well-balanced methods in the sense of Greenberg-Leroux [28]. In the present setting, this means that the prescribed drops are exactly restored for time-independent discrete solutions. Both methods rely on a two numerical fluxes approach, the difference of the latter approximates the mass of the Dirac source term. The first procedure is in the spirit of a well-balanced strategy due to Gosse [27] and extends to the present setting numerical methods developed by Ambroso et al. [3,4] within the frame of coupling technics with transmission conditions. It is based on ghost state reconstructions at the coupling interface $x=0$ that allow to use any existing two states numerical fluxes. The side effect of this flexibility is a poor resolution of the prescribed pressure drops for general Riemann initial data, despite that the method is exact in case of pairs of states that are perfectly at rest. To improve the accuracy, we propose a second method built from a convenient relaxation approximation of the original PDEs. Relaxation Riemann solutions involve a standing wave modeling the coupling interface but by contrast with exact ones, their derivation turns to be fairly simple. The main contribution of paper is to show how to handle the underlying relaxation mechanism at the coupling standing wave so as to define two numerical fluxes at the interface whose difference exactly restores the prescribed mass of the Dirac source term, whatever the Riemann initial data is. In other words, the proposed relaxation method imposes the required conservation property of the mass flow and the prescribed deviation from it concerning the momentum for both unsteady and steady solutions.

The model is presented in Section 2. In Section 4, we describe the two numerical methods and discuss their respective merits in the resolution of the prescribed singular pressure drops. In Section 5, numerical illustrations clearly highlight the distinctive properties of both methods and strongly support the interest of the proposed relaxation approach.

\section{Presentation of the Coupling Problem}

\subsection{Statement of the PDE problem}

We consider the flow of a barotropic gas in one space dimension. We denote $x \in \mathbb{R}$ the space variable whereas $t>0$ denotes time. The space domain $\mathbb{R}$ is separated by an infinitely thin interface $\mathcal{I}$ located at $x=0$, across which the flow may experience singular pressure losses. For reasons put forward along the Introduction, the flow dynamics is modeled on each side of the interface by the isentropic Euler equations but closed with distinct equations of state: namely, the pressure law $p(\tau)$, where $\tau$ is the specific volume, differs across the interface and shifts from $p_{L}(\tau)$ for $x<0$ to $p_{R}(\tau)$ for $x>0$. The PDE model under consideration can be written as:

$$
\partial_{t} \mathbf{u}+\partial_{x} \mathbf{f}(\mathbf{u}, x)=\mathcal{M}(t) \delta_{x=0}, \quad t>0, \quad x \in \mathbb{R},
$$

where the unknown $\mathbf{u}=(\rho, \rho u)^{T}$, with $\rho$ the density of the fluid and $u$ its velocity, belongs to the following natural phase space

$$
\omega=\left\{\mathbf{u}=(\rho, \rho u) \in \mathbb{R}^{2}, \rho>0, \rho u \in \mathbb{R}\right\} .
$$

The non-homogeneous flux-function $\mathbf{f}: \omega \times \mathbb{R} \rightarrow \mathbb{R}^{2}$ reads

$$
\mathbf{f}(\mathbf{u}, x)=\left(\begin{array}{c}
\rho u \\
\rho u^{2}+p(\tau, x)
\end{array}\right), \quad p(\tau, x)= \begin{cases}p_{L}(\tau), & x<0 \\
p_{R}(\tau), & x>0\end{cases}
$$


It will be convenient hereafter to use the following condensed notations:

$$
\mathbf{f}(\mathbf{u}, x)= \begin{cases}\mathbf{f}_{L}(\mathbf{u}), & x<0 \\ \mathbf{f}_{R}(\mathbf{u}), & x>0\end{cases}
$$

Here, the pressure laws $p_{\alpha}(\tau)$ with $\alpha=L, R$ are assumed to obey $p_{\alpha}^{\prime}(\tau)<0$ and $p_{\alpha}^{\prime \prime}(\tau)>0$ for all $\tau>0$ : namely the underlying first order systems in (2.1) for $x<0$ and $x>0$ are strictly hyperbolic over the phase space $\omega$. The characteristic fields of the two systems are genuinely non-linear and each system has two distinct eigenvalues respectively given by:

$$
\lambda_{\alpha}^{ \pm}(\mathbf{u})=u \pm c_{\alpha}(\tau), \quad c_{\alpha}(\tau)=\tau \sqrt{-p_{\alpha}^{\prime}(\tau)}, \quad \alpha=L, R .
$$

At last, the singular source term in (2.1) where $\delta_{x=0}$ refers to the Dirac measure concentrated at $x=0$, models the singular pressure drops at the interface $x=0$ and its mass $\mathcal{M}(t)$ reads:

$$
\mathcal{M}(t)=\left(\begin{array}{c}
\mathcal{M}_{\rho}(t) \\
\mathcal{M}_{\rho u}(t)
\end{array}\right)=\left(\begin{array}{c}
0 \\
\mathcal{M}_{\rho u}(t)
\end{array}\right), \quad t>0
$$

As expected, the conservation of the density $\rho$ holds for all times in contrast with the momentum $\rho u$ which departure from conservation is dictated by the singular loss $\mathcal{M}_{\rho u}(t) \delta_{x=0}$. An exhaustive description of semiempirical laws can be found in Idel'cik [34] or Miller [37]. For simplicity in the present work, the load $\mathcal{M}_{\rho u}(t)$ is assumed to be a given function to time.

Being prescribed some initial data $\mathbf{u}_{0}: \mathbb{R} \rightarrow \omega$, solving the associated Cauchy problem for (2.1) amounts to find a function $\mathbf{u}: \mathbb{R} \times \mathbb{R}_{+} \rightarrow \omega$ solution of:

$$
\begin{aligned}
& \partial_{t} \mathbf{u}+\partial_{x} \mathbf{f}_{L}(\mathbf{u})=0, \quad t>0, \quad x<0, \\
& \partial_{t} \mathbf{u}+\partial_{x} \mathbf{f}_{R}(\mathbf{u})=0, \quad t>0, \quad x>0,
\end{aligned}
$$

and subject to the following jump conditions across the interface $x=0$ :

$$
\mathbf{f}_{R}\left(\mathbf{u}\left(0^{+}, t\right)\right)-\mathbf{f}_{L}\left(\mathbf{u}\left(0^{-}, t\right)\right)=\mathcal{M}(t), \quad t>0 .
$$

Clearly the above jump relations can be understood as a set of conditions to couple solutions of $(2.5)$ in $x<0$ and (2.6) in $x>0$. When the load $\mathcal{M}(t)$ identically vanishes, the resulting coupling is conservative but in many situations of interest we have put forward along the Introduction, $\mathcal{M}(t)$ departs from zero.

Of central importance in the present work is the Cauchy problem for (2.1) with a prescribed constant load $\mathcal{M}=\left(0, \mathcal{M}_{\rho u}\right)^{T}$ and initial data under the form:

$$
\mathbf{u}_{0}(x)= \begin{cases}\mathbf{u}_{L}, & x<0 \\ \mathbf{u}_{R}, & x>0\end{cases}
$$

where $\mathbf{u}_{L}$ and $\mathbf{u}_{R}$ are two given states in $\omega$. The expected solution is easily seen to be self-similar and its knowledge (or an approximation of it) can serve within the usual finite volume framework to approximate the solution of (2.1) for general initial data $\mathbf{u}_{0}$ and time dependent Dirac source term $\mathcal{M}(t) \delta_{x=0}$ over successive time slabs $(t, t+\Delta t)$ with $\Delta t$ small enough. A central question then concerns existence and uniqueness of a self-similar solution of (2.5)-(2.7) for a prescribed initial data (2.8) and a given constant load $\mathcal{M}$. To address this issue, it is worth observing that the present setting actually falls with a larger framework for non-linear hyperbolic equations under the non-conservation form:

$$
\left\{\begin{array}{l}
\partial_{t} \mathbf{u}+\partial_{x} \mathbf{f}(\mathbf{u}, a)-\mathbf{g}(\mathbf{u}) \partial_{x} a=0 \\
\partial_{t} a=0
\end{array}\right.
$$


where $a$ is a scalar unknown determined by its initial data $a_{0}(x)$ while $\mathbf{g}: \omega \rightarrow \mathbb{R}^{2}$ is a given smooth function. The study of such PDE models has been pioneered by Isaacson-Temple [29] and continued by several authors, the reader is referred for instance to Goatin-LeFloch [23] and the references therein. A straightforward connection with our setting is obtained when considering

$$
a_{0}(x)=\left\{\begin{array}{l}
0, x<0 \\
1, x>0
\end{array}, \quad \mathbf{g}(\mathbf{u})=\mathcal{M} .\right.
$$

Existence of solutions of the Riemann problem for (2.9) can be obtained $[23,29]$ under fairly general assumptions on $\mathbf{f}$ and $\mathbf{g}$. These results readily apply to the present setting for $\left|\mathbf{u}_{R}-\mathbf{u}_{L}\right|,|\mathcal{M}|$ sufficiently small and pressure laws $p_{L}, p_{R}$ close enough. Besides mandatory entropy conditions that stay beyond the scope of the present paper (see $[23,29]$ for the details), uniqueness holds unless non-linear waves exhibit vanishing speeds of propagation within the Riemann fan. Interactions in between non-linear waves with vanishing velocities and the linearly degenerate standing wave associated with $a$ result in the so-called resonance phenomena for which multiple self-similar solutions may be built under generic assumptions on $\mathbf{f}, \mathbf{g}$ and some natural monotonicity property on $a_{0}(x)$. This situation is expected to hold true in our setting since in the one hand the eigenvalues $(2.3)$ of the non-linear waves for the left and right hyperbolic systems (2.5)-(2.6) may well vanish while in the second hand, due to the strict convexity of each of the pressure law $p_{\alpha}(\tau)$, the jump conditions $(2.7)$ at $x=0$ :

$$
\left\{\begin{array}{l}
(\rho u)_{+}-(\rho u)_{-}=0 \\
\left(\rho u^{2}+p_{R}(\tau)\right)_{+}-\left(\rho u^{2}+p_{L}(\tau)\right)_{-}=\mathcal{M}_{\rho u},
\end{array}\right.
$$

may admit for a given left trace $\mathbf{u}_{-}$, either zero, one or two solutions $\mathbf{u}_{+}$depending on the amplitude of $\left|\mathcal{M}_{\rho u}\right|$. The reported two sets of properties may interfere in a negative way to give birth to multiple self-similar solutions along the same vein of [29]. Observe indeed that the present situation is quite similar to the one encountered in the frame of the shallow water equations for which $p_{L}(\tau)=p_{R}(\tau)=g /\left(2 \tau^{2}\right)$ ( $g$ being the gravity constant) while $\mathcal{M}_{\rho u}$ reflects a jump in the bathymetry at $x=0$ (see [23] for instance).

This being said, let us again emphasize that the present work is concerned with nuclear thermal hydraulics where the flows of interest exhibit rather small Mach numbers [8], namely the speed of propagation of the non-linear waves in (2.1) are mostly dominated by the sound speed and thus keeps values away from zero. The reported resonance phenomena is of artificial interest and the jump conditions (2.10) admit generically two branches of solutions: namely a subsonic and a supersonic one. We always choose the subsonic branch, restricting ourselves to subsonic coupled solutions of (2.5)-(2.7) for which uniqueness holds true. Observe that considering two subsonic states $\mathbf{u}_{L}$ and $\mathbf{u}_{R}$ in (2.8), namely such that

$$
\left|\frac{u_{L}}{c_{L}\left(\tau_{L}\right)}\right|<1, \quad\left|\frac{u_{R}}{c_{R}\left(\tau_{R}\right)}\right|<1,
$$

the range of loads $\mathcal{M}$ that ensures subsonicity within the whole Riemann fan obviously depends on $\mathbf{u}_{L}$ and $\mathbf{u}_{R}$, the pressure laws $p_{\alpha}(\tau), \alpha=L, R$, being fixed. Its derivation has not been addressed in the present work since this would have required the explicit knowledge of the self-similar solution of $(2.5)-(2.7)$ which calculation is cumbersome due to the non-linearities in the PDEs.

To bypass non-linearities, our main purpose is to provide relevant approximations of exact subsonic selfsimilar solutions. In particular, we stress at this stage that the subsonic admissible range of $\mathcal{M}$ is actually entirely determined for some relevant relaxation approximation of the original PDEs in Section 4.2.2. Next and besides natural stability requirements (preservation of the phase space $\omega$ ), we intend to sharply resolve the jump relations (2.7) at the interface $x=0$. We will systematically require the approximate Riemann solutions to coincide with the exact ones in the case of pairs $\left(\mathbf{u}_{L}, \mathbf{u}_{R}\right)$ giving birth to equilibrium subsonic solutions in the sense of:

Definition 2.1. Let $\mathcal{M}$ be a constant weight and $\mathbf{u}_{L}, \mathbf{u}_{R}$ two subsonic constant states (2.11) such that

$$
\mathbf{f}_{R}\left(\mathbf{u}_{R}\right)-\mathbf{f}_{L}\left(\mathbf{u}_{L}\right)=\mathcal{M},
$$


then the function

$$
\mathbf{u}(x, t)= \begin{cases}\mathbf{u}_{L}, & x<0, \quad t>0, \\ \mathbf{u}_{R}, & x>0, \quad t>0,\end{cases}
$$

is said to be an equilibrium subsonic solution of (2.1).

Preserving exactly those equilibrium solutions naturally yields well-balanced methods in the sense of LerouxGreenberg [28] for approximating the solutions of the Cauchy problem for (2.1).

\section{Well-BAlAnCED Finite VOlume METHOdS}

We propose two well-balanced numerical methods based on a two numerical fluxes strategy at the interface $x=0$. Their difference approximates the prescribed load $\mathcal{M}$ for general pairs of states $\left(\mathbf{u}_{L}, \mathbf{u}_{R}\right)$ and restores the latter exactly in case of pairs verifying the equilibrium property (2.12). The first method relies on ghost state reconstructions as pioneered by Glimm et al. [22] for the separation of fluid components across an interface. A pair of ghost states is reconstructed from the states of the Riemann problem at the interface, so as to mimic the expected jump relations (2.7). This technic has been proved powerful in a series a works by Ambroso et al. [3,4] devoted to the numerical coupling of hyperbolic systems based on transmission conditions (see also [5]). We show how to extend the ghost state reconstruction in the present coupling setting, in the spirit of Gosse [26] for hyperbolic systems with source terms (see also Bouchut [9]). Its main advantage is its flexibility: it can be applied to any existing two states numerical fluxes. The method is by construction well- balanced for pairs of states verifying (2.12). Its main drawback is that for more general pairs, mass conservation is not exactly verified while the approximate pressure loss departs from the exact one $\mathcal{M}_{\rho u}$. This well-known drawback already holds in a purely conservative setting [22]. To circumvent this shortcoming, we propose a second well-balanced method based on a dedicated approximate Riemann solver built from a relaxation approximation of the original PDEs (2.1). The approximate Riemann fan involves a standing wave modeling the interface $x=0$ and across which a convenient relaxation version of the jump relations (2.7) are prescribed. In contrast with the original PDEs, the resulting approximate Riemann solution is fairly easily derived. In addition, it naturally yields left and right numerical fluxes at $x=0$ whose difference exactly restores the prescribed load $\mathcal{M}=\left(0, \mathcal{M}_{\rho u}\right)$ entering the jump relations (2.7) whatever is the pair of left and right states $\left(\mathbf{u}_{L}, \mathbf{u}_{R}\right)$ at the interface. This is the very novelty of the present paper.

\subsection{General numerical standpoint}

This paragraph introduces the required numerical notations. We denote $\Delta t$ the time step, $\Delta x$ the space step and $\nu=\Delta t / \Delta x$ their ratio. The interfaces of the cells are located in $x_{j}=j \Delta x$ for $j \in \mathbb{Z}$ so that the interface $x=0$ at which the Dirac source term acts comes with index $j=0$. Intermediate times are $t^{n}=n \Delta t$ for $n \in \mathbb{N}$. We seek at each time $t^{n}$ a piecewise constant approximate solution $x \rightarrow \mathbf{u}_{\nu}\left(x, t^{n}\right)$ of the solution $\mathbf{u}$ of the Cauchy problem (2.1) with initial data $\mathbf{u}_{0}$ :

$$
\mathbf{u}_{\nu}\left(x, t^{n}\right)=\mathbf{u}_{j+1 / 2}^{n} \quad \text { for } \quad x \in C_{j+1 / 2}=\left[x_{j}, x_{j+1}\right),
$$

defining at $t=0$ :

$$
\mathbf{u}_{j+1 / 2}^{0}=\frac{1}{\Delta x} \int_{x_{j}}^{x_{j+1}} \mathbf{u}_{0}(x) \mathrm{d} x, j \in \mathbb{Z} .
$$

For simplicity, we use a 3-point finite-volume method to update the discrete solution $\mathbf{u}_{\nu}\left(x, t^{n}\right)$ at time $t^{n+1}$ :

$$
\begin{aligned}
\mathbf{u}_{j-1 / 2}^{n+1} & =\mathbf{u}_{j-1 / 2}^{n}-\nu\left(\left(\mathbf{g}_{L}\right)_{j}^{n}-\left(\mathbf{g}_{L}\right)_{j-1}^{n}\right), & & j \leq 0, \\
\mathbf{u}_{j+1 / 2}^{n+1} & =\mathbf{u}_{j+1 / 2}^{n}-\nu\left(\left(\mathbf{g}_{R}\right)_{j+1}^{n}-\left(\mathbf{g}_{R}\right)_{j}^{n}\right), & & j \geq 0 .
\end{aligned}
$$


For indexes $j<0$ (respectively $j>0$ ), numerical fluxes $\left(\mathbf{g}_{L}\right)_{j}^{n}$ (respectively $\left(\mathbf{g}_{R}\right)_{j}^{n}$ ), are computed from any given locally Lipschitz continuous numerical flux function $\mathbf{g}_{L}: \omega \times \omega \rightarrow \mathbb{R}^{2}$ that is consistent with the exact flux $\mathbf{f}_{L}$ (resp. $\mathbf{g}_{R}: \omega \times \omega \rightarrow \mathbb{R}^{2}$ consistent with $\mathbf{f}_{R}$ ), namely:

$$
\mathbf{g}_{\alpha}(\mathbf{u}, \mathbf{u})=\mathbf{f}_{\alpha}(\mathbf{u}), \quad \text { for all } \mathbf{u} \in \omega, \quad \alpha=L, R,
$$

and are given by:

$$
\begin{array}{ll}
\left(\mathbf{g}_{L}\right)_{j}^{n}=\mathbf{g}_{L}\left(\mathbf{u}_{j-1 / 2}^{n}, \mathbf{u}_{j+1 / 2}^{n}\right), & j<0, \\
\left(\mathbf{g}_{R}\right)_{j}^{n}=\mathbf{g}_{R}\left(\mathbf{u}_{j-1 / 2}^{n}, \mathbf{u}_{j+1 / 2}^{n}\right), & j>0 .
\end{array}
$$

At the coupling interface labelled by index $j=0$, the updating formulas (3.1)-(3.2) make use of two distinct numerical fluxes $\left(\mathbf{g}_{L}\right)_{0}^{n}$ and $\left(\mathbf{g}_{R}\right)_{0}^{n}$, approximating respectively $\mathbf{f}_{L}\left(\mathbf{u}\left(0^{-}, t\right)\right)$ and $\mathbf{f}_{R}\left(\mathbf{u}\left(0^{+}, t\right)\right)$. Their definition must be related to the prescribed weight $\mathcal{M}(t)$ and they will be derived in order to provide some approximation of the jump conditions (2.7) at each time $t^{n}$ :

$$
\left(\mathbf{g}_{R}\right)_{0}^{n}-\left(\mathbf{g}_{L}\right)_{0}^{n} \approx \mathcal{M}^{n}, \quad \mathcal{M}^{n}=\frac{1}{\Delta t} \int_{t_{n}}^{t_{n+1}} \mathcal{M}(t) d t, \quad n>0 .
$$

At last, the ratio $\nu$ is chosen under a classical CFL restriction. In the next section, we describe two methods to compute the numerical fluxes $\left(\mathbf{g}_{L}\right)_{0}^{n}$ and $\left(\mathbf{g}_{R}\right)_{0}^{n}$, and we discuss in which sense the discrete jump conditions (3.4) is achieved.

\section{TWO NUMERICAL FLUXES APPROACHES}

\subsection{Ghost state reconstructions}

The first method we present relies on ghost state reconstructions at the interface $x=0$ so as to define the numerical fluxes $\left(\mathbf{g}_{L}\right)_{0}^{n}$ and $\left(\mathbf{g}_{R}\right)_{0}^{n}$ from any given existing consistent numerical flux functions $\mathbf{g}_{L}$ and $\mathbf{g}_{R}$.

Being given $\mathbf{u}^{L}\left(\mathbf{u}_{1 / 2}^{n}\right.$ and $\left.\mathcal{M}^{n}\right)$, we consider the following non-linear algebraic problem in the unknown $\mathbf{u}^{L}\left(\mathbf{u}_{1 / 2}^{n}, \mathcal{M}^{n}\right)$ :

$$
\mathbf{f}_{R}\left(\mathbf{u}_{1 / 2}^{n}\right)-\mathbf{f}_{L}\left(\mathbf{u}^{L}\left(\mathbf{u}_{1 / 2}^{n}, \mathcal{M}^{n}\right)\right)=\mathcal{M}^{n}
$$

Since we assume subsonic discrete solutions and as already discussed in the previous section, the proposed equations generically admits two solutions: a subsonic and a supersonic one. We choose the subsonic solution to define the ghost state $\mathbf{u}^{L}\left(\mathbf{u}_{1 / 2}^{n}, \mathcal{M}^{n}\right)$ and we introduce:

$$
\left(\mathbf{g}_{L}\right)_{0}^{n}=\mathbf{g}_{L}\left(\mathbf{u}_{-1 / 2}^{n}, \mathbf{u}^{L}\left(\mathbf{u}_{1 / 2}^{n}, \mathcal{M}^{n}\right)\right)
$$

where $\mathbf{g}_{L}: \omega \times \omega \rightarrow \mathbb{R}^{2}$ denotes ones favorite numerical flux fonction, consistent with $\mathbf{f}_{L}(3.3)$. In a symmetric way, to define $\left(\mathbf{g}_{R}\right)_{0}^{n}$, the weight $\mathcal{M}^{n}$ is addressed when solving for the ghost state $\mathbf{u}^{R}\left(\mathbf{u}_{-1 / 2}^{n}, \mathcal{M}^{n}\right)$ the next system of equations:

$$
\mathbf{f}_{R}\left(\mathbf{u}^{R}\left(\mathbf{u}_{-1 / 2}^{n}, \mathcal{M}^{n}\right)\right)-\mathbf{f}_{L}\left(\mathbf{u}_{-1 / 2}^{n}\right)=\mathcal{M}^{n}
$$

so as to introduce

$$
\left(\mathbf{g}_{R}\right)_{0}^{n}=\mathbf{g}_{R}\left(\mathbf{u}^{R}\left(\mathbf{u}_{-1 / 2}^{n}, \mathcal{M}^{n}\right), \mathbf{u}_{1 / 2}^{n}\right)
$$


Due to the strict convexity of each of the pressure law $p_{\alpha}, \alpha=L, R$, it can be seen that the nonlinear algebraic problems (4.1) and (4.3) to be solved admit either zero, or two solutions depending on the amplitude of $\left|\mathcal{M}^{n}\right|$, the states $\mathbf{u}_{-1 / 2}^{n}$ and $\mathbf{u}_{1 / 2}^{n}$ being fixed. In practice, the left and right fluxes $\mathbf{f}_{L}$ and $\mathbf{f}_{R}$ do not depart too much so that expected values of the weight generically yield two solutions for each problem: namely a subsonic and a supersonic solution.

Motivated by our multiphase flow applications, we always choose the subsonic solutions. The following statement assesses the validity of the so-called two-Riemann problem method.

Lemma 4.1. Let be given two subsonic states $\mathbf{u}_{-}$and $\mathbf{u}_{+}$in $\omega$ and a constant load $\mathcal{M}$ such that:

$$
\mathbf{f}_{R}\left(\mathbf{u}_{+}\right)-\mathbf{f}_{L}\left(\mathbf{u}_{-}\right)=\mathcal{M}
$$

Then the subsonic solution $\mathbf{u}^{L}\left(\mathbf{u}_{+}, \mathcal{M}\right)$ of equation (4.1) (respectively $\mathbf{u}^{R}\left(\mathbf{u}_{-}, \mathcal{M}\right)$ of equation (4.3)) exists and coincides with $\mathbf{u}_{-}$(respectively with $\mathbf{u}_{+}$). Using any given pair of consistent numerical fluxes (3.3), the following identities are in order:

$$
\begin{aligned}
\mathbf{g}_{L}\left(\mathbf{u}_{-}, \mathbf{u}^{L}\left(\mathbf{u}_{+}, \mathcal{M}\right)\right) & =\mathbf{f}_{L}\left(\mathbf{u}_{-}\right), \\
\mathbf{g}_{R}\left(\mathbf{u}^{R}\left(\mathbf{u}_{-}, \mathcal{M}\right), \mathbf{u}_{+}\right) & =\mathbf{f}_{R}\left(\mathbf{u}_{+}\right) .
\end{aligned}
$$

Therefore, the two-Riemann problem method (4.1), (4.2) and (4.3), (4.4) preserves stationary solutions of the coupling problem (2.1).

Proof. By definition, the states $\mathbf{u}^{L}$ and $\mathbf{u}^{R}$ under consideration are the subsonic solutions of

$$
\begin{aligned}
\mathbf{f}_{R}\left(\mathbf{u}_{+}\right)-\mathbf{f}_{L}\left(\mathbf{u}^{L}\left(\mathbf{u}_{+}, \mathcal{M}\right)\right) & =\mathcal{M}, \\
\mathbf{f}_{R}\left(\mathbf{u}^{R}\left(\mathbf{u}_{-}, \mathcal{M}\right)\right)-\mathbf{f}_{L}\left(\mathbf{u}_{-}\right) & =\mathcal{M} .
\end{aligned}
$$

By $(4.5), \mathbf{u}^{L}\left(\mathbf{u}_{+}, \mathcal{M}\right)=\mathbf{u}_{-}$and $\mathbf{u}^{R}\left(\mathbf{u}_{-}, \mathcal{M}\right)=\mathbf{u}_{+}$are the solutions we seek for. The rest of the proof follows immediately from the definitions (4.2)-(4.4) of the two consistent numerical fluxes at the interface $x=0$.

\subsection{A Relaxation model approach}

In this section, we propose a coupling method based on a Relaxation approach for the approximation of the hyperbolic system (2.1). We refer the reader to [9] and [35] for general discussions on the Relaxation approach in the approximation of hyperbolic systems of conservation laws. Our discussion is divided in two subsections. At first, we will show how to solve the Riemann problem for the Relaxation model associated to the coupling problem in the case of the conservative coupling model (i.e. when $\mathcal{M}(t)$ completely vanishes in problem $(2.1)$ ). Then, the second subsection will treat the case of the non-conservative coupling problem (i.e. with $\mathcal{M}(t) \neq 0$ ) that is the approximation by the Relaxation model of problem (2.1).

\subsubsection{Conservative coupling by the Relaxation method}

We begin this section by writing the equations (2.1) closed by condition $\mathcal{M}(t)=0$, with initial data $\mathbf{u}_{0}(x)$ :

$$
\left\{\begin{array}{l}
\partial_{t} \rho+\partial_{x}(\rho u)=0, \\
\partial_{t}(\rho u)+\partial_{x}\left(\rho u^{2}+p(\tau, x)\right)=0, \quad t>0, \quad x \in \mathbb{R}
\end{array}\right.
$$

where

$$
p(\tau, x)= \begin{cases}p_{L}(\tau), & x<0, \\ p_{R}(\tau), & x>0 .\end{cases}
$$


The present conservative coupling requires the continuity of the exact fluxes at the interface $x=0$ :

$$
\begin{aligned}
\rho u\left(0^{-}, t\right) & =\rho u\left(0^{+}, t\right), \\
\left(\rho u^{2}+p_{L}(\tau)\right)\left(0^{-}, t\right) & =\left(\rho u^{2}+p_{R}(\tau)\right)\left(0^{+}, t\right) .
\end{aligned}
$$

We propose to approximate the solutions of (4.6) by those of the following relaxation system

$$
\left\{\begin{array}{l}
\partial_{t} \rho_{\lambda}+\partial_{x}(\rho u)_{\lambda}=0 \\
\partial_{t}(\rho u)_{\lambda}+\partial_{x}\left(\rho u^{2}+\pi\right)_{\lambda}=0 \\
\partial_{t}(\rho \pi)_{\lambda}+\partial_{x}\left(\rho \pi u+a^{2} u\right)_{\lambda}=\lambda \rho_{\lambda}(p(\tau, x)-\pi)_{\lambda}, \quad t>0, \quad x \in \mathbb{R},
\end{array}\right.
$$

where $\lambda>0$ plays the role of a relaxation rate. Indeed in (4.9), observe that the original non-homogenous pressure law $p(\tau, x)$ is replaced by a new unknown denoted by $\pi_{\lambda}$. The solutions of the original equations (4.6) are formally restored in the limit of an infinite relaxation parameter $\lambda \rightarrow+\infty$, since we formally get in this limit

$$
\lim _{\lambda \rightarrow+\infty} \pi_{\lambda}=p(\tau, x)
$$

from the last equation in (4.9). Then, the given real number $a>0$ in (4.9) can be understood as a frozen value of $\rho c(\tau, x)$ where $c(\tau, x)=\tau \sqrt{-\partial_{\tau} p(\tau, x)}$ is the sound speed (see [9] for instance). It is known that to prevent the relaxation procedure from instabilities in the regime of a large relaxation parameter $\lambda \gg 1$, the frozen value $a>0$ in (4.9) has to be chosen large enough (see for instance [9] and Prop. 4.2 below for a precise definition). The relaxation system (4.9) is given the following condensed form:

$$
\partial_{t} \mathbf{U}_{\lambda}+\partial_{x} \mathbf{F}\left(\mathbf{U}_{\lambda}\right)=\lambda \mathbf{S}\left(\mathbf{U}_{\lambda}, x\right), \quad x \in \mathbb{R}, \quad t>0,
$$

with

$$
\mathbf{U}=\left(\begin{array}{c}
\rho \\
\rho u \\
\rho \pi
\end{array}\right), \quad \mathbf{F}(\mathbf{U})=\left(\begin{array}{c}
\rho u \\
\rho u^{2}+\pi \\
\rho \pi u+a^{2} u
\end{array}\right), \quad \mathbf{S}(\mathbf{U}, x)=\left(\begin{array}{c}
0 \\
0 \\
\rho(p(\tau, x)-\pi)
\end{array}\right)
$$

We define the new space $\Omega$ of states $\mathbf{U}$ :

$$
\Omega=\left\{\mathbf{U}=(\rho, \rho u, \rho \pi) \in \mathbb{R}^{3}, \rho>0, \rho u \in \mathbb{R}, \rho \pi \in \mathbb{R}\right\} .
$$

Let us underline that in the present relaxation framework, the flux function $\mathbf{F}(\mathbf{U})$ does not depend on the space variable $x$. In fact, the spatial non-homogeneity of the original flux is taken into account when always considering well-prepared initial data $\mathbf{U}_{0}$ for (4.10), namely such that the relaxation pressure $\pi_{0}$ is systematically set at equilibrium:

$$
\pi_{0}(x)=p\left(\tau_{0}(x), x\right)=\left\{\begin{array}{l}
p_{L}\left(\tau_{0}(x)\right), x<0 \\
p_{R}\left(\tau_{0}(x)\right), x>0
\end{array}\right.
$$

The basic properties of system (4.10) are as follows. Being given $a>0$, it admits three distinct real eigenvalues:

$$
\lambda_{1}(\mathbf{U})=u-a \tau, \quad \lambda_{2}(\mathbf{U})=u, \quad \lambda_{3}(\mathbf{U})=u+a \tau,
$$

and is thus strictly hyperbolic over the phase space (4.11). A strong property is that all the associated fields are linearly degenerate [9].

Let us now describe the numerical approximation of the solutions of (4.6) by means of those of (4.9). Assuming a discrete solution $\mathbf{u}_{\nu}\left(x, t^{n}\right)$ of system (4.6) at time $t^{n}$, this one is evolved to the next time level $t^{n+1}$ 


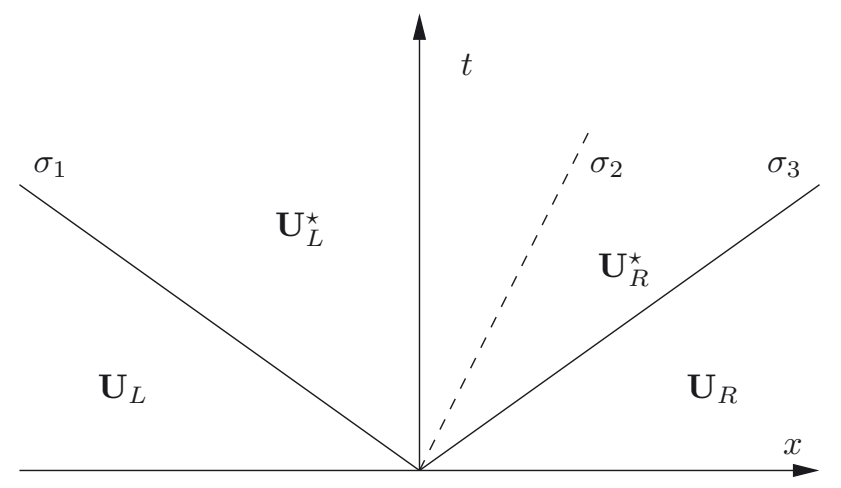

FiguRE 1. Generic wave pattern in the Riemann solution for the conservative coupling relaxation model.

into two steps. These steps can be understood in terms of a splitting strategy for (4.9), choosing first $\lambda=0$ and then considering the limit of an infinite relaxation parameter $\lambda \rightarrow \infty$.

First step: time evolution $\left(t^{n} \rightarrow t^{n+1-}\right)$

In the first step, we set $\lambda=0$ in (4.10) in order to solve for $t \in[0, \Delta t], \Delta t$ small enough, the following homogeneous initial value problem

$$
\left\{\begin{array}{l}
\partial_{t} \mathbf{U}+\partial_{x} \mathbf{F}(\mathbf{U})=0, \quad x \in \mathbb{R}, \quad t>0 \\
\mathbf{U}(x, 0)=\mathbf{U}_{\nu}\left(x, t^{n}\right),
\end{array}\right.
$$

with

$$
\mathbf{U}_{\nu}\left(x, t^{n}\right)=\left(\mathbf{u}_{\nu}\left(x, t^{n}\right),(\rho \pi)_{\nu}\left(x, t^{n}\right)\right)^{T} .
$$

Here we recall that $\rho \pi$ is set at equilibrium:

$$
(\rho \pi)_{\nu}\left(x, t^{n}\right)=\rho\left(x, t^{n}\right) p\left(\tau_{\nu}\left(x, t^{n}\right), x\right) .
$$

The frozen velocity $a^{n}>0$ is prescribed at time $t^{n}$ so as to satisfy the following Whitham like stability condition (see [9] for instance)

$$
a^{n}>a_{\#}\left(\mathbf{U}\left(x, t^{n}\right)\right) \equiv \max _{x \in \mathbf{R}}\left(\sqrt{-\partial_{\tau} p\left(\tau\left(x, t^{n}\right), x\right)}\right),
$$

a precise definition will be given in Proposition 4.2 below. Under the usual CFL restriction

$$
\frac{\Delta t}{\Delta x} \max _{x \in \mathbb{R}}\left(\left|u_{\nu}\left(x, t^{n}\right)\right|+a^{n} \tau_{\nu}\left(x, t^{n}\right)\right) \leq \frac{1}{2}
$$

the solution of (4.14) is classically built from a sequence of non-interacting Riemann solutions for the homogeneous relaxation system. Hence being given two states $\mathbf{U}_{L}, \mathbf{U}_{R}$ in $\Omega$, the self-similar solution of

$$
\left\{\begin{array}{l}
\partial_{t} \mathbf{U}+\partial_{x} \mathbf{F}(\mathbf{U})=0, \quad x \in \mathbb{R}, \quad t>0, \\
\mathbf{U}(x, 0)= \begin{cases}\mathbf{U}_{L}, & x<0, \\
\mathbf{U}_{R}, & x>0,\end{cases}
\end{array}\right.
$$

we denote $\mathcal{W}\left(x / t ; \mathbf{U}_{L}, \mathbf{U}_{R}\right)$, is generically made of four constant states $\mathbf{U}_{L}, \mathbf{U}_{L}^{\star}, \mathbf{U}_{R}^{\star}$ and $\mathbf{U}_{R}$ systematically separated by contact discontinuities propagating with velocity $\sigma_{i}, i=\{1,2,3\}$ (see Fig. 1). 
The precise definition of the intermediate states is the matter of the following statement:

Proposition 4.2. Being given two states $\mathbf{U}_{L}$ and $\mathbf{U}_{R}$ in the phase space $\Omega$, let us prescribe a frozen velocity a according to

$$
a\left(\mathbf{U}_{L}, \mathbf{U}_{R}\right)>\max \left(a_{\#}\left(\mathbf{U}_{L}, \mathbf{U}_{R}\right), a_{\star}\left(\mathbf{U}_{L}, \mathbf{U}_{R}\right)\right),
$$

where $a_{\#}\left(\mathbf{U}_{L}, \mathbf{U}_{R}\right)$ is defined in (4.16) and

$$
a_{\star}\left(\mathbf{U}_{L}, \mathbf{U}_{R}\right)=\frac{\left(u_{R}-u_{L}\right)+\sqrt{\left(u_{R}-u_{L}\right)^{2}+4 \max \left(\tau_{L}, \tau_{R}\right)\left|\pi_{R}-\pi_{L}\right|}}{2 \min \left(\tau_{L}, \tau_{R}\right)} .
$$

Then the Riemann problem (4.18) admits a unique self-similar solution $\mathcal{W}\left(x / t ; \mathbf{U}_{L}, \mathbf{U}_{R}\right)$ for which the intermediate states $\mathbf{U}_{L}^{\star}$ and $\mathbf{U}_{R}^{\star}$ belong to $\Omega$ with

$$
\begin{aligned}
& u^{\star}\left(\mathbf{U}_{L}, \mathbf{U}_{R}\right)=u_{L}^{\star}=u_{R}^{\star}=\frac{1}{2}\left(u_{L}+u_{R}\right)-\frac{1}{2 a}\left(\pi_{R}-\pi_{L}\right), \\
& \pi^{\star}\left(\mathbf{U}_{L}, \mathbf{U}_{R}\right)=\pi_{L}^{\star}=\pi_{R}^{\star}=\frac{1}{2}\left(\pi_{L}+\pi_{R}\right)-\frac{a}{2}\left(u_{R}-u_{L}\right), \\
& \tau_{L}^{\star}\left(\mathbf{U}_{L}, \mathbf{U}_{R}\right)=\tau_{L}+\frac{1}{a}\left(u^{\star}-u_{L}\right)>0, \\
& \tau_{R}^{\star}\left(\mathbf{U}_{L}, \mathbf{U}_{R}\right)=\tau_{R}+\frac{1}{a}\left(u_{R}-u^{\star}\right)>0 .
\end{aligned}
$$

Proof. The proof relies on simple calculations but since some of the main ingredients will be used in the next section, we briefly sketch it to be self-contained. The contact discontinuities are moving with the characteristic speed $\lambda_{i}$ of the corresponding field:

$$
\sigma_{1}=\lambda_{1}\left(\mathbf{U}_{L}\right)=\lambda_{1}\left(\mathbf{U}_{L}^{\star}\right), \quad \sigma_{2}=\lambda_{2}\left(\mathbf{U}_{L}^{\star}\right)=\lambda_{2}\left(\mathbf{U}_{R}^{\star}\right), \quad \sigma_{3}=\lambda_{3}\left(\mathbf{U}_{R}^{\star}\right)=\lambda_{3}\left(\mathbf{U}_{R}\right) .
$$

Using these identities together with the usual Rankine-Hugoniot relations for (4.18) easily yield the following jump conditions

$$
\begin{aligned}
& a\left(u_{L}^{\star}-u_{L}\right)+\left(\pi_{L}^{\star}-\pi_{L}\right)=0, \\
& u_{L}^{\star}=u_{R}^{\star} \equiv u^{\star}, \\
& \pi_{L}^{\star}=\pi_{R}^{\star} \equiv \pi^{\star}, \\
& a\left(u_{R}^{\star}-u_{R}\right)+\left(\pi_{R}-\pi_{R}^{\star}\right)=0 .
\end{aligned}
$$

Easy manipulations left to the reader give the formulas for $u^{\star}$ and $\pi^{\star}$. Then, the intermediate specific volumes $\tau_{L}^{\star}$ and $\tau_{R}^{\star}$ are recovered from the first and last identities in (4.25). The positivity requirements $\tau_{L}^{\star}>0$ and $\tau_{R}^{\star}>0$ are easily seen to be equivalent to the natural wave ordering condition

$$
\sigma_{1}<\sigma_{2}<\sigma_{3}
$$

which is satisfied provided that the frozen velocity $a$ is chosen according to (4.19).

\section{Second step: relaxation $\left(t^{n+1-} \rightarrow t^{n+1}\right)$}

The solution obtained after this convective step is noted $\mathbf{U}_{\nu}\left(x, t^{n+1-}\right)$. In the second step, we solve the following system of ordinary differential equations

$$
\left\{\begin{array}{l}
\partial_{t} \rho=0 \\
\partial_{t}(\rho u)=0 \\
\partial_{t}(\rho \pi)=\lambda \rho(p(\tau, x)-\pi),
\end{array}\right.
$$


with $\lambda \rightarrow+\infty$ and initial condition given by the solution of the first step $\mathbf{U}_{\nu}\left(x, t^{n+1-}\right)$. Finally the discrete solution $\mathbf{u}_{\nu}\left(x, t^{n+1}\right)$ corresponds to

$$
\mathbf{U}_{\nu}\left(x, t^{n+1}\right)=\left(\mathbf{u}_{\nu}\left(x, t^{n+1}\right),(\rho \pi)_{\nu}\left(x, t^{n+1}\right)\right)^{T}
$$

with

$$
\begin{aligned}
\mathbf{u}_{\nu}\left(x, t^{n+1}\right) & =\mathbf{u}_{\nu}\left(x, t^{n+1-}\right) \\
(\rho \pi)_{\nu}\left(x, t^{n+1}\right) & =\rho\left(x, t^{n+1-}\right) p\left(\tau_{\nu}\left(x, t^{n+1-}\right), x\right) .
\end{aligned}
$$

To summarize this paragraph, we give the detailed form of the numerical fluxes $\left(\mathbf{g}_{L}\right)_{0}^{n}$ and $\left(\mathbf{g}_{R}\right)_{0}^{n}$ entering equations (3.1) and (3.2). At time $t^{n}$, being given the states $\mathbf{u}_{-1 / 2}^{n}$ and $\mathbf{u}_{1 / 2}^{n}$, we define $\mathbf{U}_{-1 / 2}^{n}=$ $\left(\mathbf{u}_{-1 / 2}^{n}, \rho_{-1 / 2}^{n} p_{L}\left(\tau_{-1 / 2}^{n}\right)\right)$ and $\mathbf{U}_{1 / 2}^{n}=\left(\mathbf{u}_{1 / 2}^{n}, \rho_{1 / 2}^{n} p_{R}\left(\tau_{1 / 2}^{n}\right)\right)$ according to equation (4.15). The required fluxes $\left(\mathbf{g}_{L}\right)_{0}^{n}$ and $\left(\mathbf{g}_{R}\right)_{0}^{n}$ are recovered from the first two components of $\mathbf{F}\left(\mathcal{W}\left(0^{+} ; \mathbf{U}_{-1 / 2}^{n}, \mathbf{U}_{1 / 2}^{n}\right)\right)$ setting

$$
\left(\mathbf{g}_{L}\right)_{0}^{n}=\left(\mathbf{g}_{R}\right)_{0}^{n}=\left(\begin{array}{c}
\rho u \\
\rho u^{2}+\pi
\end{array}\right)\left(\mathcal{W}\left(0^{+} ; \mathbf{U}_{-1 / 2}^{n}, \mathbf{U}_{1 / 2}^{n}\right)\right) .
$$

\subsubsection{Non conservative coupling by the relaxation method}

The frame of a non-zero Dirac source term $\mathcal{M} \delta_{x=0}$ in (2.1) is more involved than the setting of a conservative coupling. We show that the direct extension of the relaxation model (4.9) to the present framework allows for a fairly simple derivation of approximate self-similar solutions with suitable well-balanced and positivity properties. With the notations of the previous section, the Riemann relaxation coupling problem writes:

$$
\left\{\begin{array}{l}
\partial_{t} \mathbf{U}+\partial_{x} \mathbf{F}(\mathbf{U})=\widetilde{\mathcal{M}} \delta_{x=0}, \quad x \in \mathbb{R}, \quad t>0, \\
\mathbf{U}(x, 0)= \begin{cases}\mathbf{U}_{L}, & x<0, \\
\mathbf{U}_{R}, & x>0,\end{cases}
\end{array}\right.
$$

where $\widetilde{\mathcal{M}}$ is built from $\mathcal{M}=\left(0, \mathcal{M}_{\rho u}\right)$ in $(2.1)$ when setting

$$
\widetilde{\mathcal{M}}=\left(\begin{array}{c}
\mathcal{M} \\
\mathcal{M}_{\rho \pi}
\end{array}\right)=\left(\begin{array}{c}
0 \\
\mathcal{M}_{\rho u} \\
\mathcal{M}_{\rho \pi}
\end{array}\right)
$$

Here, the additional weight $\mathcal{M}_{\rho \pi}$ acting on the relaxation pressure equation of system (4.9) may seem arbitrary but actually it must be given a precise non zero definition in order to exactly capture the equilibrium solutions of the original coupling problem (2.1) (see Prop. (4.3) below).

Let us briefly comment on the structure of the self-similar solution of the relaxation coupling Riemann problem (4.32). In contrast with the last paragraph, a non-trivial additional wave with zero speed - the socalled coupling standing wave - has to be dealt with. This supplementary wave accounts for the Dirac source term $\widetilde{\mathcal{M}} \delta_{x=0}$ and yields the left and right traces of self-similar solutions at the interface to obey the jump conditions:

$$
\begin{aligned}
& (\rho u)_{+}-(\rho u)_{-}=0, \\
& \left(\rho u^{2}+\pi\right)_{+}-\left(\rho u^{2}+\pi\right)_{-}=\mathcal{M}_{\rho u}, \\
& \left(\rho \pi u+a^{2} u\right)_{+}-\left(\rho \pi u+a^{2} u\right)_{-}=\mathcal{M}_{\rho \pi} .
\end{aligned}
$$

As already put forward, we only address the case of subsonic self-similar solutions of (4.32). It means that being given two states $\mathbf{U}_{L}$ and $\mathbf{U}_{R}$ satisfying $\left|u_{L} / a \tau_{L}\right|<1$ and $\left|u_{R} / a \tau_{R}\right|<1$ (see indeed the sub-characteristic condition (4.16)), we focus ourselves on Riemann solutions with subsonic left and right traces at $x=0$ :

$$
\left|\frac{u_{-}}{a \tau_{-}}\right|=\left|\frac{u_{+}}{a \tau_{+}}\right|=\left|\frac{m}{a}\right|<1
$$




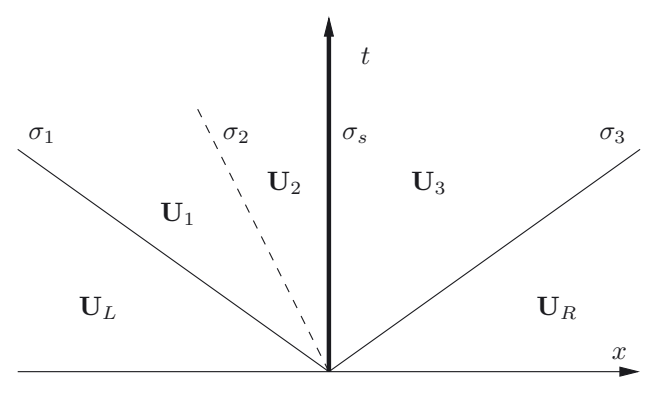

(a) Case $\sigma_{2}<0$

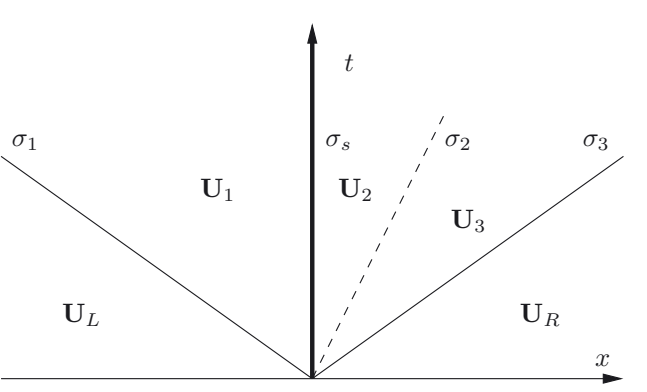

(b) Case $\sigma_{2}>0$

Figure 2. Wave patterns of subsonic solutions of the coupled Riemann problem (4.32).

where we have set from the first equation in (4.34)

$$
m \equiv \rho_{-} u_{-}=\rho_{+} u_{+} .
$$

Hence, the typical wave patterns of the self-similar solutions under consideration are displayed in Figure 2. Obviously the expected self-similar solution of (4.32) depends on the given data $\mathbf{U}_{L}, \mathbf{U}_{R}$ but also on the two prescribed weights $\mathcal{M}_{\rho u}$ and $\mathcal{M}_{\rho \pi}$. A pair of subsonic states $\left(\mathbf{U}_{L}, \mathbf{U}_{R}\right)$ being fixed, arbitrary values of those weights may result in one hand in a wave pattern distinct from the ones depicted in Figure 2, and in a second hand in states within the wave fan with a non-positive density $\rho$.

\subsubsection{Subsonic admissible domain for $\left(\mathcal{M}_{\rho \mathrm{u}}, \mathcal{M}_{\rho \pi}\right)$ and Riemann solution}

The values of $\mathcal{M}_{\rho u}$ and $\mathcal{M}_{\rho \pi}$ must be suitably prescribed in order to fit with the expected wave structures while guaranteeing the positivity of all the intermediate densities. We will see hereafter that the latter positivity requirement is closely related to the natural wave ordering condition $\sigma_{1}<\sigma_{2}<\sigma_{3}$, as already put forward in the proof of Proposition 4.2. The next statement provides a sharp characterization of the relevant weights $\mathcal{M}_{\rho u}, \mathcal{M}_{\rho \pi}$ built from the eigenvalues $\lambda_{1}\left(U_{L}\right), \lambda_{3}\left(U_{R}\right)$ in (4.13) and the intermediate velocities $u^{\star}\left(\mathbf{U}_{L}, \mathbf{U}_{R}\right)$ defined in (4.23), which we write for short $u^{\star}$. In addition, the proposed statement shows that the sign of $\sigma_{2}$, namely the relative position of the $\lambda_{2^{-}}$wave with respect to the coupling standing one, can be entirely determined from the pair $\left(\mathcal{M}_{\rho u}, \mathcal{M}_{\rho \pi}\right)$ and the given states $\mathbf{U}_{L}, \mathbf{U}_{R}$.

Proposition 4.3. Let be given two subsonic states $\mathbf{U}_{L}$ and $\mathbf{U}_{R}$ in $\Omega$, then there exists a non-empty open convex domain $\mathcal{D}_{\text {adm }}\left(\mathbf{U}_{L}, \mathbf{U}_{R}\right) \subset \mathbb{R}_{\mathcal{M}_{\rho u}} \times \mathbb{R}_{\mathcal{M}_{\rho \pi}}$ such that for any given pair $\left(\mathcal{M}_{\rho u}, \mathcal{M}_{\rho \pi}\right) \in \mathcal{D}_{a d m}\left(\mathbf{U}_{L}, \mathbf{U}_{R}\right)$ the Riemann problem (4.32) admits a unique self-similar subsonic solution with positive intermediate densities.

In addition, the so-called admissible domain $\mathcal{D}_{a d m}\left(\mathbf{U}_{L}, \mathbf{U}_{R}\right)$ is the union of two open convex sets $\mathcal{D}_{\text {adm }}^{-}\left(\mathbf{U}_{L}, \mathbf{U}_{R}\right)$ and $\mathcal{D}_{a d m}^{+}\left(\mathbf{U}_{L}, \mathbf{U}_{R}\right)$ respectively given by

$$
\begin{aligned}
\mathcal{D}_{a d m}^{-}\left(\mathbf{U}_{L}, \mathbf{U}_{R}\right)= & \left\{\left(\mathcal{M}_{\rho u}, \mathcal{M}_{\rho \pi}\right) \in \mathbb{R}^{2} /-2 a^{2} \lambda_{3}\left(\mathbf{U}_{R}\right)<a \mathcal{M}_{\rho u}+\mathcal{M}_{\rho \pi}<-2 a^{2} u^{\star},\right. \\
& -2 a^{2} u^{\star}\left(2 u^{\star}-\lambda_{3}\left(\mathbf{U}_{R}\right)\right)<\left(2 u^{\star}-\lambda_{3}\left(\mathbf{U}_{R}\right)\right) a \mathcal{M}_{\rho u}+\lambda_{3}\left(\mathbf{U}_{R}\right) \mathcal{M}_{\rho \pi} \\
< & -2 a^{2} u^{\star}\left(2 \lambda_{1}\left(\mathbf{U}_{L}\right)\left(\lambda_{3}\left(\mathbf{U}_{R}\right)-u^{\star}\right)+u^{\star}\left(2 u^{\star}-\lambda_{3}\left(\mathbf{U}_{R}\right)\right)\right\},
\end{aligned}
$$

and

$$
\begin{aligned}
\mathcal{D}_{a d m}^{+}\left(\mathbf{U}_{L}, \mathbf{U}_{R}\right)= & \left\{\left(\mathcal{M}_{\rho u}, \mathcal{M}_{\rho \pi}\right) \in \mathbb{R}^{2} /-2 a^{2} u^{\star}<a \mathcal{M}_{\rho u}+\mathcal{M}_{\rho \pi}<-2 a^{2} \lambda_{1}\left(\mathbf{U}_{L}\right),\right. \\
& -2 a^{2} u^{\star}\left(2 u^{\star}-\lambda_{1}\left(\mathbf{U}_{L}\right)\right)<\left(2 u^{\star}-\lambda_{1}\left(\mathbf{U}_{L}\right)\right) a \mathcal{M}_{\rho u}-\lambda_{1}\left(\mathbf{U}_{L}\right) \mathcal{M}_{\rho \pi} \\
< & -2 a^{2} u^{\star}\left(2 \lambda_{3}\left(\mathbf{U}_{R}\right)\left(\lambda_{1}\left(\mathbf{U}_{L}\right)-u^{\star}\right)+u^{\star}\left(2 u^{\star}-\lambda_{1}\left(\mathbf{U}_{L}\right)\right)\right\},
\end{aligned}
$$


with the property that if $\left(\mathcal{M}_{\rho u}, \mathcal{M}_{\rho \pi}\right) \in \mathcal{D}_{\text {adm }}^{-}\left(\mathbf{U}_{L}, \mathbf{U}_{R}\right)$ (respectively $\left(\mathcal{M}_{\rho u}, \mathcal{M}_{\rho \pi}\right) \in \mathcal{D}_{a d m}^{+}\left(\mathbf{U}_{L}, \mathbf{U}_{R}\right)$ ) then the self-similar solution of (4.32) verifies $\sigma_{2}<0$ (resp. $\sigma_{2}>0$ ) and thus corresponds to the wave pattern exhibited in Figure $2 a$ (resp. (2b)). At last, the set $\overline{\mathcal{D}_{\text {adm }}^{-}}\left(\mathbf{U}_{L}, \mathbf{U}_{R}\right) \cap \overline{\mathcal{D}_{a d m}^{+}}\left(\mathbf{U}_{L}, \mathbf{U}_{R}\right)$ reads $\left\{\left(-2 u^{\star}, 0\right)\right\}$ and corresponds to the limiting case of a vanishing speed $\sigma_{2}=0$.

The proof of the proposed statement relies on the following technical result.

Lemma 4.4. Let be given a pair of subsonic states $\left(\mathbf{U}_{L}, \mathbf{U}_{R}\right)$ and a pair of weights $\left(\mathcal{M}_{\rho u}, \mathcal{M}_{\rho \pi}\right)$. Assuming a subsonic self-similar solution of (4.32) in the sense of (4.37) with positive intermediate densities, such a solution is uniquely determined and can be written in the case $\sigma_{2}<0$ :

$$
\mathcal{W}\left(x / t ; \mathcal{M}_{\rho u}, \mathcal{M}_{\rho \pi}, \mathbf{U}_{L}, \mathbf{U}_{R}\right)=\left\{\begin{array}{l}
\mathbf{U}_{L}, x / t<\lambda_{1}\left(\mathbf{U}_{L}\right) \\
\mathbf{U}_{1}, \lambda_{1}\left(\mathbf{U}_{L}\right)<x / t<\lambda_{2}\left(\mathbf{U}_{1}\right), \\
\mathbf{U}_{-}, \lambda_{2}\left(\mathbf{U}_{1}\right)<x / t<0 \\
\mathbf{U}_{+}, 0<x / t<\lambda_{3}\left(\mathbf{U}_{R}\right), \\
\mathbf{U}_{R}, \lambda_{3}\left(\mathbf{U}_{R}\right)<x / t
\end{array}\right.
$$

and in the case $\sigma_{2}>0$ :

$$
\mathcal{W}\left(x / t ; \mathcal{M}_{\rho u}, \mathcal{M}_{\rho \pi}, \mathbf{U}_{L}, \mathbf{U}_{R}\right)=\left\{\begin{array}{l}
\mathbf{U}_{L}, x / t<\lambda_{1}\left(\mathbf{U}_{L}\right) \\
\mathbf{U}_{-}, \lambda_{1}\left(\mathbf{U}_{L}\right)<x / t<0 \\
\mathbf{U}_{+}, 0<x / t<\lambda_{2}\left(\mathbf{U}_{3}\right) \\
\mathbf{U}_{3}, \lambda_{2}\left(\mathbf{U}_{3}\right)</ t<\lambda_{3}\left(\mathbf{U}_{R}\right), \\
\mathbf{U}_{R}, \lambda_{3}\left(\mathbf{U}_{R}\right)<x / t
\end{array}\right.
$$

Assuming $\sigma_{2}<0$, we have:

$$
m=\frac{a \mathcal{M}_{\rho u}+\mathcal{M}_{\rho \pi}+2 a^{2} u^{\star}}{2 a^{2} \tau_{R}^{\star}}<0, \quad u_{1}=u_{+}, \quad \tau_{1}=\tau_{L}-\frac{1}{a}\left(u_{L}-u_{-}\right), \quad \pi_{1}=\pi_{-},
$$

where $m$ is the mass flux defined in (4.38) while when $\sigma_{2}>0$ :

$$
m=\frac{a \mathcal{M}_{\rho u}-\mathcal{M}_{\rho \pi}+2 a^{2} u^{\star}}{2 a^{2} \tau_{L}^{\star}}>0, \quad u_{3}=u_{+}, \quad \tau_{3}=\tau_{R}+\frac{1}{a}\left(u_{R}-u_{+}\right), \quad \pi_{3}=\pi_{+} .
$$

The left and right traces of the solution at the interface $x=0$ read

$$
\begin{array}{lll}
u_{-}=u^{\star}+\frac{a \mathcal{M}_{\rho u}-\mathcal{M}_{\rho \pi}}{2 a(a-m)}, & \tau_{-}=\frac{u_{-}}{m}, & \pi_{-}=\pi^{\star}-\frac{2 a \mathcal{M}_{\rho u}-\mathcal{M}_{\rho \pi}}{2(a-m)}, \\
u_{+}=u^{\star}+\frac{a \mathcal{M}_{\rho u}+\mathcal{M}_{\rho \pi}}{2 a(a+m)}, & \tau_{+}=\frac{u_{+}}{m}, & \pi_{+}=\pi^{\star}+\frac{2 a \mathcal{M}_{\rho u}+\mathcal{M}_{\rho \pi}}{2(a+m)} .
\end{array}
$$

At last, assuming $\sigma_{2}=0$, the self-similar solution comes with $m=0$ and writes

$$
\mathcal{W}\left(x / t ; \mathcal{M}_{\rho u}, \mathcal{M}_{\rho \pi}, \mathbf{U}_{L}, \mathbf{U}_{R}\right)=\left\{\begin{array}{l}
\mathbf{U}_{L}, x / t<\lambda_{1}\left(\mathbf{U}_{L}\right), \\
\mathbf{U}_{-}, \lambda_{1}\left(\mathbf{U}_{L}\right)<x / t<0, \\
\mathbf{U}_{+}, 0<x / t<\lambda_{3}\left(\mathbf{U}_{R}\right), \\
\mathbf{U}_{R}, \lambda_{3}\left(\mathbf{U}_{R}\right)<x / t
\end{array}\right.
$$

with

$$
\begin{aligned}
& u_{-}=0, \tau_{-}=\tau_{L}-\frac{u_{L}}{a}, \pi_{-}=\pi^{\star}+a u^{\star}, \\
& u_{+}=0, \tau_{+}=\tau_{R}-\frac{u_{R}}{a}, \pi_{+}=\pi^{\star}-a u^{\star} .
\end{aligned}
$$


Proof. The proof is derived in the case $\sigma_{2}<0$. Similar steps are involved in the situations $\sigma_{2}>0, \sigma_{2}=0$ and are left to the reader. Let us thus assume a subsonic wave pattern as depicted in Figure 2a. Let us first observe the identities $u_{1}=u_{-}$and $\pi_{1}=\pi_{-}$inferred from the property that both the velocity $u$ and the pressure $\pi$ are the two Riemann invariants of the $\lambda_{2}$-wave. A straightforward adaptation of arguments given in the proof of Proposition 4.2 then provides the following jump conditions, respectively for the first and last wave:

$$
\begin{aligned}
& a\left(u_{-}-u_{L}\right)+\left(\pi_{-}-\pi_{L}\right)=0, \\
& a\left(u_{+}-u_{R}\right)+\left(\pi_{R}-\pi_{+}\right)=0 .
\end{aligned}
$$

Considering the mass flux $m$ introduced in (4.38), the last two jump relations at the coupling interface (4.34) can recast:

$$
\begin{aligned}
& m\left(u_{+}-u_{-}\right)+\left(\pi_{+}-\pi_{-}\right)=\mathcal{M}_{\rho u}, \\
& m\left(\pi_{+}-\pi_{-}\right)+a^{2}\left(u_{+}-u_{-}\right)=\mathcal{M}_{\rho \pi} .
\end{aligned}
$$

Understanding in a first step $m$ as a free parameter, equations (4.53) and (4.55) read as a $4 \times 4$ linear system in the unknown $\left(u_{-}, \pi_{-}, u_{+}, \pi_{+}\right)$. This linear system is seen to be invertible provided that $a^{2}-m^{2} \neq 0$, such a condition is met since by assumption $|m / a|<1$ (see indeed (4.37)). Easy calculations then yield the expected definitions (4.49)-(4.50) of the intermediate velocities $u_{ \pm}$and pressures $\pi_{ \pm}$. The specific volumes $\tau_{-}$and $\tau_{+}$ follow from the definition of the mass flux $m$ while $\tau_{1}$ is inferred from the identity $\lambda_{1}\left(\mathbf{U}_{1}\right)=\lambda_{L}\left(\mathbf{U}_{L}\right)$. Let us now determine the mass flux $m$ entering the proposed formulas. In that aim, observe that the property $\lambda_{3}\left(\mathbf{U}_{+}\right)=\lambda_{3}\left(\mathbf{U}_{R}\right)$ once multiplied by $\rho_{+}$writes $m+a=\lambda_{3}\left(\mathbf{U}_{R}\right) \rho_{+}$, so that one gets:

$$
(a+m) u_{+}=\lambda_{3}\left(\mathbf{U}_{R}\right) \rho_{+} u_{+}=\lambda_{3}\left(\mathbf{U}_{R}\right) m .
$$

The definition of $u_{+}$in (4.50) yields $2 a(a+m)\left(u_{+}-u^{\star}\right)=a \mathcal{M}_{\rho u}+\mathcal{M}_{\rho \pi}$ and easy algebraic manipulations give from (4.57):

$$
2 a\left(\lambda_{3}\left(\mathbf{U}_{R}\right)-u^{\star}\right) m=a \mathcal{M}_{\rho u}+\mathcal{M}_{\rho \pi}+2 a^{2} u^{\star} .
$$

The desired expression (4.47) for $m$ follows from the identity $\lambda_{3}\left(\mathbf{U}_{R}\right)=\lambda_{3}\left(\mathbf{U}_{R}^{\star}\right)$ established in (4.25). We have thus established that any given subsonic self-similar solution corresponding to the wave pattern displayed in Figure 2a is uniquely determined by the proposed formulas (4.47)-(4.50).

Equipped with Lemma 4.4, let us prove Proposition 4.3.

Proof. We derive the admissible subset $\mathcal{D}_{\text {adm }}^{-}\left(\mathbf{U}_{L}, \mathbf{U}_{R}\right)$ assuming $u_{-}=\sigma_{2}<0$. Similar steps are in order concerning $\mathcal{D}_{\text {adm }}^{-}\left(\mathbf{U}_{L}, \mathbf{U}_{R}\right)$ when $\sigma_{2}>0$. Let us first qualify pairs of weights $\left(\mathcal{M}_{\rho u}, \mathcal{M}_{\rho \pi}\right)$ so that the selfsimilar solution exhibited in Lemma 4.4 is subsonic with a negative velocity $u_{-}$. Assuming in a first step a positive specific volume $\tau_{-}>0$, this amounts to impose

$$
-a<m<0,
$$

with a mass flux $m$ given by (4.47), that is to say after multiplication by $2 a^{2} \tau_{R}^{\star}$ while using the identity $\lambda_{3}\left(\mathbf{U}_{R}^{\star}\right)=\left(\mathbf{U}_{R}\right)$ established in $(4.25)$ :

$$
-2 a^{2} \lambda_{3}\left(\mathbf{U}_{R}\right)<a \mathcal{M}_{\rho u}+\mathcal{M}_{\rho \pi}<-2 a^{2} u^{\star} .
$$

The proposed bounds are the first set of inequalities entering the definition of $\mathcal{D}_{\text {adm }}^{-}\left(\mathbf{U}_{L}, \mathbf{U}_{R}\right)$. Let us now require a negative intermediate velocity $u_{-}$, namely from its definition in (4.49):

$$
u^{\star}+\frac{a \mathcal{M}_{\rho u}-\mathcal{M}_{\rho \pi}}{2 a(a-m)}<0,
$$


and equivalently from the fact that $a-m>0$ in view of (4.59):

$$
2 a(a-m) u^{\star}<-a \mathcal{M}_{\rho u}+\mathcal{M}_{\rho \pi} .
$$

Noticing from (4.47) the identity

$$
2 a(a-m)=-\frac{1}{a \tau_{R}^{\star}}\left(a \mathcal{M}_{\rho u}+\mathcal{M}_{\rho \pi}+2 a^{2}\left(u^{\star}-a \tau_{R}^{\star}\right)\right), \quad \text { with } u^{\star}-a \tau_{R}^{\star}=2 u^{\star}-\lambda_{3}\left(\mathbf{U}_{R}\right),
$$

the inequality (4.62) rewrites

$$
-2 a^{2} u^{\star}\left(2 u^{\star}-\lambda_{3}\left(\mathbf{U}_{R}\right)\right)<\left(2 u^{\star}-\lambda_{3}\left(\mathbf{U}_{R}\right)\right) a \mathcal{M}_{\rho u}+\lambda_{3}\left(\mathbf{U}_{R}\right) \mathcal{M}_{\rho \pi} .
$$

The proposed condition for admissible pairs of weights $\left(\mathcal{M}_{\rho u}, \mathcal{M}_{\rho \pi}\right)$ is the lower bound in the second set of conditions expressed in the definition of $\mathcal{D}_{\text {adm }}^{-}\left(\mathbf{U}_{L}, \mathbf{U}_{R}\right)$. Observe that under the two conditions (4.60)-(4.64), the specific volume $\tau_{-}=u_{-} / m$ is naturally positive. Next in view of the identity $\lambda_{3}\left(\mathbf{U}_{+}\right)=\lambda_{3}\left(\mathbf{U}_{R}\right)$ where by assumption $\lambda_{3}\left(\mathbf{U}_{R}\right)>0$, requiring the positivity of $\tau_{+}$is equivalent to ask for $\rho+\left(u_{+}+a \tau_{+}\right)>0$, namely $m+a>0$. But this holds true under the condition (4.60). To conclude, it suffices to express a condition for ensuring $\tau_{1}>0$. Its representation formula in (4.47) once multiplied by $a$ yields the equivalent condition: $u_{-}>u_{L}-a \tau_{L}$ which is nothing but the ordering condition $\lambda_{1}\left(\mathbf{U}_{L}\right)<\lambda_{2}\left(\mathbf{U}_{-}\right)=u_{-}$. That is to say from the definition of $u_{-}$

$$
u^{\star}+\frac{a \mathcal{M}_{\rho u}-\mathcal{M}_{\rho \pi}}{2 a(a-m)}>\lambda_{1}\left(\mathbf{U}_{L}\right) .
$$

Using once again equation (4.63), easy calculations give

$$
\begin{aligned}
-2 a^{2}\left(2 u^{\star}-\lambda_{3}\left(\mathbf{U}_{R}\right)\right)\left(\lambda_{1}\left(\mathbf{U}_{L}\right)-u^{\star}\right) & <\left(\lambda_{1}\left(\mathbf{U}_{L}\right)-\left(2 u^{\star}-\lambda_{3}\left(\mathbf{U}_{R}\right)\right)\right) a \mathcal{M}_{\rho u}+\left(\lambda_{1}\left(\mathbf{U}_{R}\right)-\lambda_{3}\left(\mathbf{U}_{R}\right)\right) \mathcal{M}_{\rho \pi} \\
& =\lambda_{1}\left(\mathbf{U}_{L}\right)\left(a \mathcal{M}_{\rho u}+\mathcal{M}_{\rho \pi}\right)-\left(\left(2 u^{\star}-\lambda_{3}\left(\mathbf{U}_{R}\right)\right) a \mathcal{M}_{\rho u}+\lambda_{3}\left(\mathbf{U}_{R}\right) \mathcal{M}_{\rho \pi}\right) .
\end{aligned}
$$

But inequalities (4.60) and $\lambda_{1}\left(\mathbf{U}_{L}\right)<0$ clearly allow to strengthen the above condition according to

$$
-2 a^{2}\left(2 u^{\star}-\lambda_{3}\left(\mathbf{U}_{R}\right)\right)\left(\lambda_{1}\left(\mathbf{U}_{L}\right)-u^{\star}\right)<-2 a^{2} \lambda_{1}\left(\mathbf{U}_{L}\right) \lambda_{3}\left(\mathbf{U}_{R}\right)-\left(\left(2 u^{\star}-\lambda_{3}\left(\mathbf{U}_{R}\right)\right) a \mathcal{M}_{\rho u}+\lambda_{3}\left(\mathbf{U}_{R}\right) \mathcal{M}_{\rho \pi}\right),
$$

which is up to a multiplication by -1 the last inequality expressed in the definition of $\mathcal{D}_{\text {adm }}^{-}\left(\mathbf{U}_{L}, \mathbf{U}_{R}\right)$. This concludes the proof.

\subsubsection{Choice of $\mathcal{M}_{\rho \pi}$ and well-balanced property of the relaxation scheme}

Motivated by the well-balanced property, we now pay a specific attention to relevant definitions of $\widetilde{\mathcal{M}}$ in $(4.33)$ from the given weight $\mathcal{M}$ in the initial value problem (2.1) such that its equilibrium solutions are exactly preserved by the relaxation approximation. More precisely, being given two subsonic states $\mathbf{u}_{L}$ and $\mathbf{u}_{R}$ in $\omega$ and a weight $\mathcal{M}_{\rho u} \in \mathbb{R}$ such that:

$$
\mathbf{f}_{R}\left(\mathbf{u}_{R}\right)-\mathbf{f}_{L}\left(\mathbf{u}_{L}\right)=\left(0, \mathcal{M}_{\rho u}\right)^{T},
$$

the weight $\mathcal{M}_{\rho \pi}$ has to be properly prescribed such that the self-similar solution of the relaxation Riemann problem (4.32) writes:

$$
\mathcal{W}\left(x / t ; \mathcal{M}_{\rho u}, \mathcal{M}_{\rho \pi}, \mathbf{U}_{L}, \mathbf{U}_{R}\right)= \begin{cases}\mathbf{U}_{L}, & x<0, \quad t>0 \\ \mathbf{U}_{R}, & x>0\end{cases}
$$


where the two constant states are defined at equilibrium:

$$
\mathbf{U}_{L}=\left(\mathbf{u}_{L}, \rho_{L} p_{L}\left(\tau_{L}\right)\right), \quad \mathbf{U}_{R}=\left(\mathbf{u}_{R}, \rho_{R} p_{R}\left(\tau_{R}\right)\right) .
$$

In other words, the self-similar solution under consideration is stationary and reduces to the initial data. Restricting such a solution to its two first components restores the next function

$$
\mathbf{u}(x, t)= \begin{cases}\mathbf{u}_{L}, & x<0, \quad t>0, \\ \mathbf{u}_{R}, & x>0,\end{cases}
$$

which is nothing the expected equilibrium solution put forward in Definition 2.1. The next statement provides the required definition of the required weight $\mathcal{M}_{\rho \pi}$ for general subsonic states $\left(\mathbf{u}_{L}, \mathbf{u}_{R}\right)$ and weight $\mathcal{M}_{\rho u}$ :

Proposition 4.5. For any given pair of subsonic states $\left(\mathbf{u}_{L}, \mathbf{u}_{R}\right)$ in $\omega^{2}$ and any given load $\mathcal{M}_{\rho u}$, let us define from the pair of equilibrium states (4.70):

$$
\mathcal{M}_{\rho \pi}^{e}\left(\mathcal{M}_{\rho u}, \mathbf{U}_{L}, \mathbf{U}_{R}\right)=m_{e}\left(\mathcal{M}_{\rho u}, \mathbf{U}_{L}, \mathbf{U}_{R}\right)\left(\mathcal{I}_{R}-\mathcal{I}_{L}\right),
$$

with

$$
\mathcal{I}_{L}=p_{L}\left(\tau_{L}\right)+a^{2} \tau_{L}, \quad \mathcal{I}_{R}=p_{R}\left(\tau_{R}\right)+a^{2} \tau_{R},
$$

and a mass flux given by

$$
m_{e}\left(\mathcal{M}_{\rho u}, \mathbf{U}_{L}, \mathbf{U}_{R}\right)=\frac{\mathcal{M}_{\rho u}+2 a u^{\star}\left(\mathbf{U}_{L}, \mathbf{U}_{R}\right)}{a\left(\tau_{L}^{\star}\left(\mathbf{U}_{L}, \mathbf{U}_{R}\right)+\tau_{R}^{\star}\left(\mathbf{U}_{L}, \mathbf{U}_{R}\right)\right)}
$$

Here the mappings $\tau_{L}^{\star}, \tau_{R}^{\star}$ and $u^{\star}$ have been introduced in Proposition 4.2. Then considering a pair of states $\left(\mathbf{u}_{L}, \mathbf{u}_{R}\right)$ such that the balance condition (4.68) holds true, the self-similar solution $\mathcal{W}\left(x / t ; \mathcal{M}_{\rho u}, \mathcal{M}_{\rho \pi}^{e}\left(\mathcal{M}_{\rho u}, \mathbf{U}_{L}, \mathbf{U}_{R}\right), \mathbf{U}_{L}, \mathbf{U}_{R}\right)$ of the Riemann problem (4.32) with initial data (4.70) coincides with (4.69).

Proof. Let us first establish in the case of a general pair of subsonic states and a given $\mathcal{M}_{\rho u}$ that a weight $\mathcal{M}_{\rho \pi}$ under the form

$$
\mathcal{M}_{\rho \pi}=m\left(\mathcal{I}_{R}-\mathcal{I}_{L}\right)
$$

with a mass flux $m$ given either by (4.47) or (4.48) necessarily writes as (4.72) with $m=m_{e}\left(\mathcal{M}_{\rho u}, \mathbf{U}_{L}, \mathbf{U}_{R}\right)$. In that aim, let us observe from the definition of the states $\mathbf{U}_{L}^{\star}$ and $\mathbf{U}_{R}^{\star}$ defined in Proposition 4.2 the following identities

$$
\mathcal{I}_{L}=\pi^{\star}+a^{2} \tau_{L}^{\star}, \quad \mathcal{I}_{R}=\pi^{\star}+a^{2} \tau_{R}^{\star} .
$$

These come from simple algebraic manipulations that are left to the reader. As a consequence, we infer

$$
\mathcal{I}_{R}-\mathcal{I}_{L}=a^{2}\left(\tau_{R}^{\star}-\tau_{L}^{\star}\right) .
$$

Equipped with this formula and considering the case of a mass flux $m$ given by (4.47) (respectively (4.48)), straightforward calculations give

$$
\left(2 a^{2} \tau_{R}^{\star}-\left(\mathcal{I}_{R}-\mathcal{I}_{L}\right)\right) m=a \mathcal{M}_{\rho u}+2 a^{2} u^{\star}, \quad\left(\operatorname{resp} .\left(2 a^{2} \tau_{L}^{\star}+\left(\mathcal{I}_{R}-\mathcal{I}_{L}\right)\right) m=a \mathcal{M}_{\rho u}+2 a^{2} u^{\star}\right),
$$

and the expected conclusion readily follows in both cases from the identity (4.77).

Let us now turn considering a pair of states $\left(\mathbf{u}_{L}, \mathbf{u}_{R}\right)$ and a weight $\mathcal{M}_{\rho u}$ verifying the balance equation (4.68) and prove that the self-similar function (4.69) is the solution $\mathcal{W}\left(x / t ; \mathcal{M}_{\rho u}, \mathcal{M}_{\rho \pi}^{e}\left(\mathcal{M}_{\rho u}, \mathbf{U}_{L}, \mathbf{U}_{R}\right), \mathbf{U}_{L}, \mathbf{U}_{R}\right)$ of the Riemann problem (4.32) with initial data (4.70). Let us first establish that the mass flux in (4.74) we write for short $m_{e}$ coincides with $m \equiv(\rho u)_{L}=(\rho u)_{R}$. The second equation in (4.68) writes

$$
\mathcal{M}_{\rho u}=m\left(u_{R}-u_{L}\right)+\left(p_{R}-p_{L}\right)=m^{2}\left(\tau_{R}-\tau_{L}\right)+\left(p_{R}-p_{L}\right),
$$


while the formulas defining $u^{\star}, \tau_{L}^{\star}$ and $\tau_{R}^{\star}$ easily yield

$$
\begin{aligned}
2 a u^{\star} & =\left(u_{R}+u_{L}\right)-\left(p_{R}-p_{L}\right)=m a\left(\tau_{R}+\tau_{L}\right)-\left(p_{R}-p_{L}\right), \\
a\left(\tau_{L}^{\star}+\tau_{R}^{\star}\right) & =a\left(\tau_{R}+\tau_{L}\right)+\left(u_{R}-u_{L}\right)=a\left(\tau_{R}+\tau_{L}\right)+m\left(\tau_{R}-\tau_{L}\right) .
\end{aligned}
$$

We thus deduce that $\mathcal{M}_{\rho u}+2 a u^{\star}=m\left(a\left(\tau_{L}^{\star}+\tau_{R}^{\star}\right)\right)$, that is to say $m_{e}=m$.

From Figure 2, it now clearly suffices to check in the case $m=m_{e}<0$, i.e. $\sigma_{2}<0$, (respectively $\sigma_{2}>0$ ) that a self-similar function defined from (4.45) (resp. (4.46)) with $\mathbf{U}_{1}=\mathbf{U}_{-}=\mathbf{U}_{L}$ and $\mathbf{U}_{+}=\mathbf{U}_{R}$ (resp. $\mathbf{U}_{-}=\mathbf{U}_{L}$ and $\mathbf{U}_{+}=\mathbf{U}_{3}=\mathbf{U}_{R}$ solves the Riemann problem (4.32) with an initial data given by (4.70). Jumps in this piecewise constant function are trivial except at the interface $x=0$ and is therefore a solution of the problem under consideration if and only if

$$
\mathbf{F}\left(\mathbf{U}_{R}\right)-\mathbf{F}\left(\mathbf{U}_{L}\right)=\left(0, \mathcal{M}_{\rho u}, \mathcal{M}_{\rho \pi}^{e}\right)^{T},
$$

with $\mathcal{M}_{\rho \pi}^{e}$ defined in (4.72). The initial data (4.70) being at equilibrium, the first two equations in (4.81) just read:

$$
\mathbf{f}_{R}\left(\mathbf{u}_{R}\right)-\mathbf{f}_{L}\left(\mathbf{u}_{L}\right)=\left(0, \mathcal{M}_{\rho u}\right)^{T},
$$

and are readily satisfied since $m_{e}=m$ while by assumption $\mathbf{u}_{L}, \mathbf{u}_{R}$ and $\mathcal{M}_{\rho u}$ solve (4.68). The left-hand side in the last equation of (4.81) writes

$$
m_{e}\left(\pi_{R}-\pi_{L}\right)+a^{2}\left(u_{R}-u_{L}\right)=m_{e}\left(\left(\pi_{R}+a^{2} \tau_{R}\right)-\left(\pi_{L}+a^{2} \tau_{L}\right)\right)
$$

since the mass flux $m_{e}=m=u_{R} / \tau_{R}=u_{L} \tau_{L}$. The right hand-side in the above identity is nothing but the definition of $\mathcal{M}_{\rho \pi}^{e}$ given in (4.72). This concludes the proof.

\subsubsection{Intermediate states and numerical fluxes at the coupling interface}

We first summarize the definition of the three intermediate states $\mathbf{U}_{1}, \mathbf{U}_{2}$ and $\mathbf{U}_{3}$ involved in the waves patterns depicted in Figure 2. In this aim, it is convenient to consider the left and right traces of the self-similar solution $\mathcal{W}\left(x / t ; m_{e}\left(\mathcal{M}_{\rho u}, \mathbf{U}_{L}, \mathbf{U}_{R}\right), \mathcal{M}_{\rho u}, \mathbf{U}_{L}, \mathbf{U}_{R}\right)$ singled out in the above statement at the interface, which we denote, respectively

$$
\mathbf{U}_{-}=\mathcal{W}\left(0^{-} ; m_{e}\left(\mathcal{M}_{\rho u}, \mathbf{U}_{L}, \mathbf{U}_{R}\right), \mathcal{M}_{\rho u}, \mathbf{U}_{L}, \mathbf{U}_{R}\right)
$$

and

$$
\mathbf{U}_{+}=\mathcal{W}\left(0^{+} ; m_{e}\left(\mathcal{M}_{\rho u}, \mathbf{U}_{L}, \mathbf{U}_{R}\right), \mathcal{M}_{\rho u}, \mathbf{U}_{L}, \mathbf{U}_{R}\right) \text {. }
$$

Observe that $m_{e} \equiv m_{e}\left(\mathcal{M}_{\rho u}, \mathbf{U}_{L}, \mathbf{U}_{R}\right)$ (which we write $m_{e}\left(\mathcal{M}_{\rho u}\right)$ for short) in (4.74) verifies $m_{e}<0$ as soon as $\sigma_{2}<0$ and $m_{e}>0$ whenever $\sigma_{2}>0$. With these solutions, observe that in Figure 2a with $\sigma_{2}<0$, we have $\mathbf{U}_{-}=\mathbf{U}_{2}$ and $\mathbf{U}_{+}=\mathbf{U}_{3}$ while by contrast in Figure $2 \mathrm{~b}$ with $\sigma_{2}>0$, we have $\mathbf{U}_{-}=\mathbf{U}_{1}$ and $\mathbf{U}_{+}=\mathbf{U}_{2}$. Equipped with the definition of the mass flux (4.74), let us introduce

$$
\mathcal{M}_{\rho \pi}^{e}\left(m_{e}\left(\mathcal{M}_{\rho u}\right), \mathbf{U}_{L}, \mathbf{U}_{R}\right)=m_{e}\left(\mathcal{M}_{\rho u}\right)\left(\mathcal{I}_{R}-\mathcal{I}_{L}\right)
$$

where we have set $\mathcal{I}_{R}=p_{R}\left(\tau_{R}\right)+a^{2} \tau_{R}$ and $\mathcal{I}_{L}=p_{L}\left(\tau_{L}\right)+a^{2} \tau_{L}$ which we write for short $\mathcal{M}_{\rho \pi}^{e}\left(\mathcal{M}_{\rho u}\right)$. Then the left and right traces $\mathbf{U}_{-}$and $\mathbf{U}_{+}$at $x=0$ of the self-similar solution displayed in Figure 2 are recovered from:

$$
\begin{array}{lll}
u_{-}=u^{\star}+\frac{a \mathcal{M}_{\rho u}-\mathcal{M}_{\rho \pi}^{e}\left(\mathcal{M}_{\rho u}\right)}{2 a\left(a-m_{e}\left(\mathcal{M}_{\rho u}\right)\right)}, & \tau_{-}=\frac{u_{-}}{m_{e}\left(\mathcal{M}_{\rho u}\right)}, & \pi_{-}=\pi^{\star}-\frac{2 a \mathcal{M}_{\rho u}-\mathcal{M}_{\rho \pi}^{e}\left(\mathcal{M}_{\rho u}\right)}{2\left(a-m_{e}\left(\mathcal{M}_{\rho u}\right)\right)}, \\
u_{+}=u^{\star}+\frac{a \mathcal{M}_{\rho u}+\mathcal{M}_{\rho \pi}^{e}\left(\mathcal{M}_{\rho u}\right)}{2 a\left(a+m_{e}\left(\mathcal{M}_{\rho u}\right)\right)}, & \tau_{+}=\frac{u_{+}}{m_{e}\left(\mathcal{M}_{\rho u}\right)}, & \pi_{+}=\pi^{\star}+\frac{2 a \mathcal{M}_{\rho u}+\mathcal{M}_{\rho \pi}^{e}\left(\mathcal{M}_{\rho u}\right)}{2\left(a+m_{e}\left(\mathcal{M}_{\rho u}\right)\right)},
\end{array}
$$


as soon as $m_{e}\left(\mathcal{M}_{\rho u}\right) \neq 0$. If $m_{e}\left(\mathcal{M}_{\rho u}\right)$ vanishes, then $\mathbf{U}_{-}=\mathbf{U}_{+}=\mathbf{U}_{L}^{\star}$ if $u^{\star}>0$ and $\mathbf{U}_{-}=\mathbf{U}_{+}=\mathbf{U}_{R}^{\star}$ otherwise (see Fig. 2). Then to conclude, we need to define $\mathbf{U}_{3}$ in the case $m_{e}\left(\mathcal{M}_{\rho u}\right)>0$ (i.e. $\left.\mathbf{U}_{1}=\mathbf{U}_{-}, \mathbf{U}_{2}=\mathbf{U}_{+}\right)$by:

$$
u_{3}=u_{+}, \quad \tau_{3}=\tau_{R}+\frac{1}{a}\left(u_{R}-u_{+}\right), \quad \pi_{3}=\pi_{+},
$$

and $\mathbf{U}_{1}$ in the case $m_{e}\left(\mathcal{M}_{\rho u}\right)<0$ (i.e. $\left.\mathbf{U}_{2}=\mathbf{U}_{-}, \mathbf{U}_{3}=\mathbf{U}_{+}\right)$by:

$$
u_{1}=u_{-}, \quad \tau_{1}=\tau_{L}-\frac{1}{a}\left(u_{L}-u_{-}\right), \quad \pi_{1}=\pi_{-} .
$$

To conclude, the expected numerical fluxes entering equations (3.1) and (3.2) are recovered as follow. At time $t^{n}$, being given the states $\mathbf{u}_{-1 / 2}^{n}$ and $\mathbf{u}_{1 / 2}^{n}$, we define $\mathbf{U}_{-1 / 2}^{n}=\left(\mathbf{u}_{-1 / 2}^{n}, \rho_{-1 / 2}^{n} p_{L}\left(\tau_{-1 / 2}^{n}\right)\right)$ and $\mathbf{U}_{1 / 2}^{n}=\left(\mathbf{u}_{1 / 2}^{n}, \rho_{1 / 2}^{n} p_{R}\left(\tau_{1 / 2}^{n}\right)\right)$ according to equation (4.15). Then from the value of $\mathcal{M}^{n}=\left(0, \mathcal{M}_{\rho u}^{n}=\right.$ $\left.\mathcal{M}_{\rho u}\left(t^{n}\right)\right)$ in (2.1) we define $\widetilde{\mathcal{M}}^{n}=\left(0, \mathcal{M}_{\rho u}^{n}, \mathcal{M}_{\rho \pi}^{e}\left(\mathcal{M}_{\rho u}^{n}\right)\right)$ with $\mathcal{M}_{\rho \pi}^{e}\left(\mathcal{M}_{\rho u}^{n}\right)$ in (4.84). The solution $\mathcal{W}\left(x / t ; m_{e}\left(\mathcal{M}_{\rho u}^{n}\right), \mathcal{M}_{\rho u}^{n}, \mathbf{U}_{-1 / 2}^{n}, \mathbf{U}_{1 / 2}^{n}\right)$ of (4.32) being deduced from the formulae (4.85) to (4.86) we have:

$$
\begin{aligned}
\left(\mathbf{g}_{L}\right)_{0}^{n} & =\left(\begin{array}{c}
\rho u \\
\rho u^{2}+\pi
\end{array}\right)\left(\mathcal{W}\left(0^{-} ; m_{e}\left(\mathcal{M}_{\rho u}^{n}\right), \mathcal{M}_{\rho u}^{n}, \mathbf{U}_{-1 / 2}^{n}, \mathbf{U}_{1 / 2}^{n}\right)\right), \\
\left(\mathbf{g}_{R}\right)_{0}^{n} & =\left(\begin{array}{c}
\rho u \\
\rho u^{2}+\pi
\end{array}\right)\left(\mathcal{W}\left(0^{+} ; m_{e}\left(\mathcal{M}_{\rho u}^{n}\right), \mathcal{M}_{\rho u}^{n}, \mathbf{U}_{-1 / 2}^{n}, \mathbf{U}_{1 / 2}^{n}\right)\right) .
\end{aligned}
$$

\section{Numerical RESUlts}

We present in this section results from numerical simulations based on the methods we propose. In the next test cases, two distinct cases of values for $\mathcal{M}_{\rho u}$ are considered. The initial condition for the flow are of Riemann type. The two constant states $\mathbf{u}_{L}$ and $\mathbf{u}_{R}$ under consideration are given by:

$$
\mathbf{u}(x, 0)= \begin{cases}\mathbf{u}_{L} & \text { if } \quad x<0 \\ \mathbf{u}_{R} & \text { if } \quad x>0\end{cases}
$$

with

\begin{tabular}{ccc}
\hline & & \\
\hline & $L$ & $R$ \\
\hline$\rho$ & 2 & 1 \\
$u$ & $u_{L}$ & 0 \\
\hline
\end{tabular}

where

$$
u_{L}=u_{R}+\sqrt{\left(p_{R}\left(\tau_{R}\right)-p_{L}\left(\tau_{L}\right)\right)\left(\tau_{L}-\tau_{R}\right)} .
$$

The pressure law is $p_{\alpha}(\tau)=\tau^{-\gamma_{\alpha}}$. The adiabatic coefficients are $\gamma_{L}=1.4$ for the left domain and $\gamma_{R}=1.6$ for the right domain. The number of mesh points is 200 and the space domain is the interval $[-0.5,0.5]$. The CFL constant is set to 0.5 .

\subsection{Conservative coupling $\mathcal{M} \equiv(0,0)$}

The purpose of the present benchmark is to illustrate the behavior of the numerical methods we have introduced to approximate the solution of problem 2.1 in the conservative setting: i.e. with $\mathcal{M}=(0,0)$. We show that the two-Riemann problem method and the relaxation strategy described in Section 4 yield discrete solutions in a fairly good agreement despite some minor discrepencies may be reported. Discrete solutions are compared on Figures 3 and 4. We observe that the approximate solution obtained by the two-Riemann problem approach exhibits a perfectly sharp discontinuity at the interface while the relaxation approach described in 


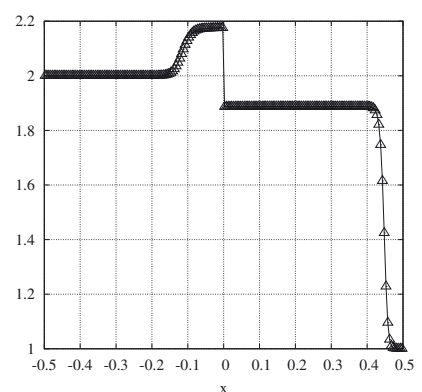

(a) Density

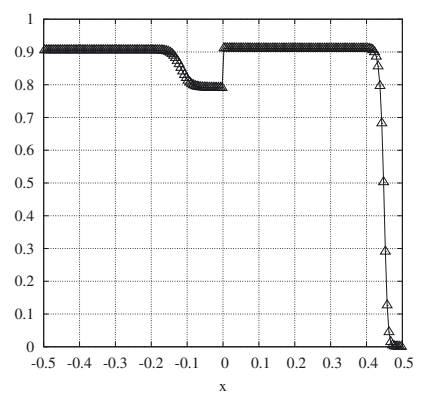

(b) Velocity

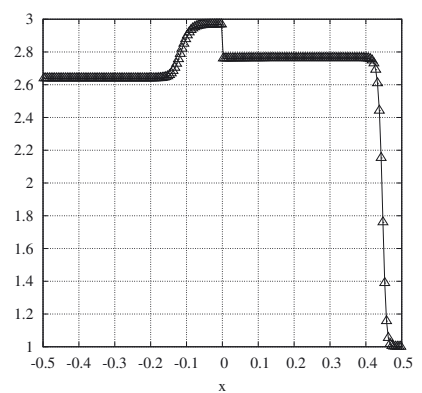

(c) Pressure

FiguRE 3. Conservative coupling: results for the two-Riemann problem method.

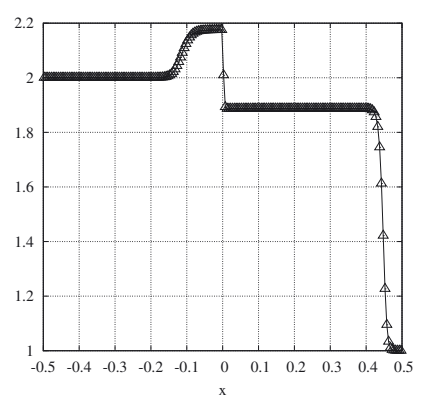

(a) Density

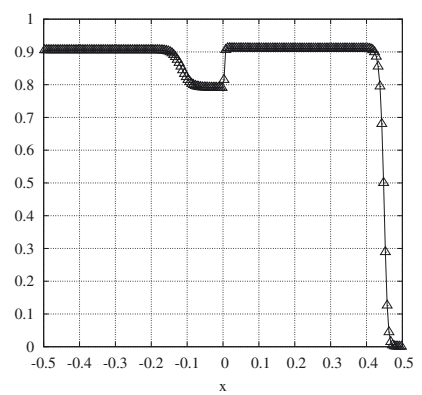

(b) Velocity

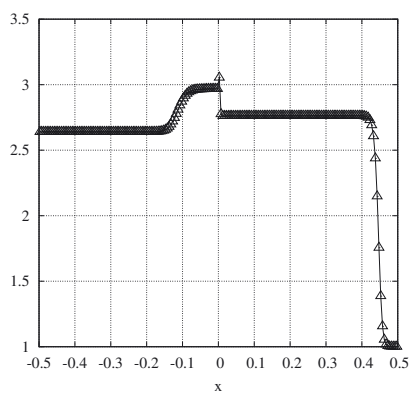

(c) Pressure

FiguRE 4. Conservative coupling: results for the Relaxation approach.

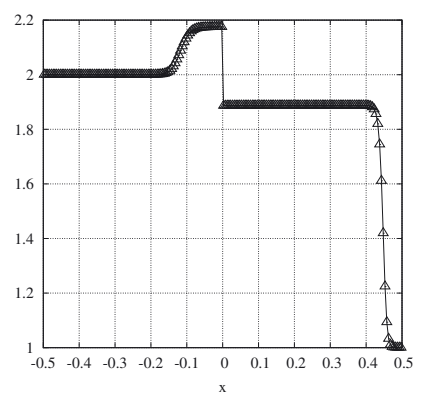

(a) Density

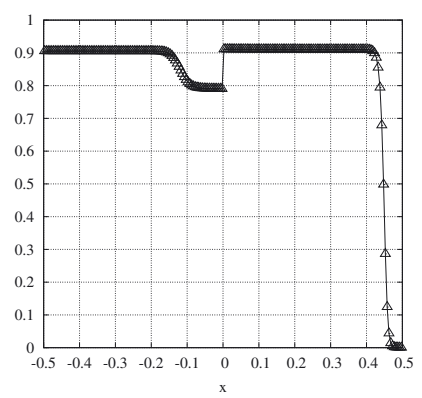

(b) Velocity

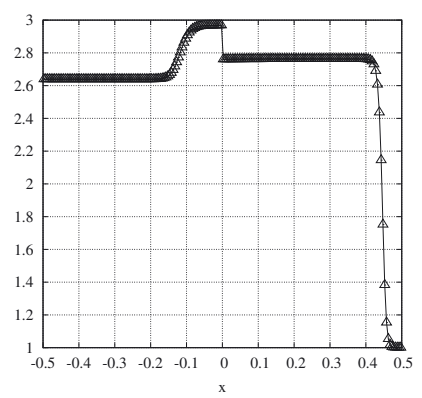

(c) Pressure

FiguRE 5. Conservative coupling: results for the Relaxation approach with weight $\mathcal{M}_{\rho \pi}^{e}$.

Section 4 displays a discrete profile with one intermediate point resulting in a slight overshoot in the pressure distribution. To go further, Figures $8 \mathrm{a}$ and $9 \mathrm{a}$ display the component of impulsion of difference $\left(\mathbf{g}_{R}\right)_{0}^{n}-\left(\mathbf{g}_{L}\right)_{0}^{n}$ in its time history and the value $\mathcal{M}_{\rho u}^{n}$ (that is here strictly zero). As expected from its design principle, the relaxation approach yields an exact balance $\left(\mathbf{g}_{R}\right)_{0}^{n}-\left(\mathbf{g}_{L}\right)_{0}^{n}=0$ at each time step. The two-Riemann problem approach achieves this exact balance after a few time steps but departure from the expected cancellation stays fairly admissible.

Figure 5 shows the results achieved by the relaxation approach described in Section 4.2 .2 with $\mathcal{M}_{\rho \pi}^{e}\left(\mathcal{M}_{\rho u}\right)$ given in (4.84) choosing $\mathcal{M}_{\rho u} \equiv 0$. Notice that the present method differs from the first relaxation strategy 


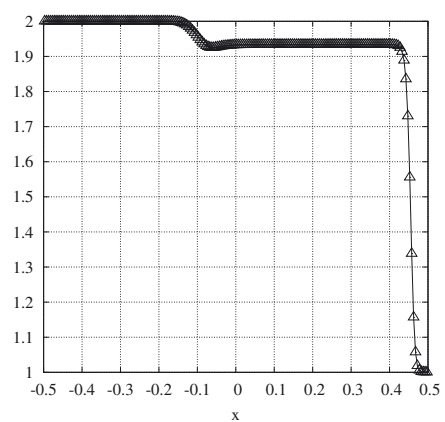

(a) Density

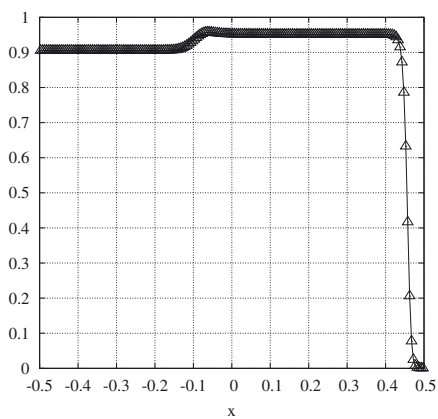

(b) Velocity

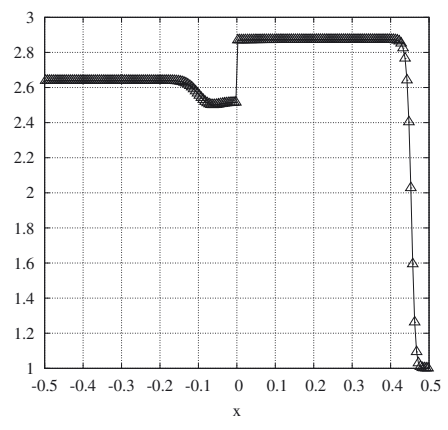

(c) Pressure

FiguRE 6. Non conservative coupling: results for the two-Riemann problem method.

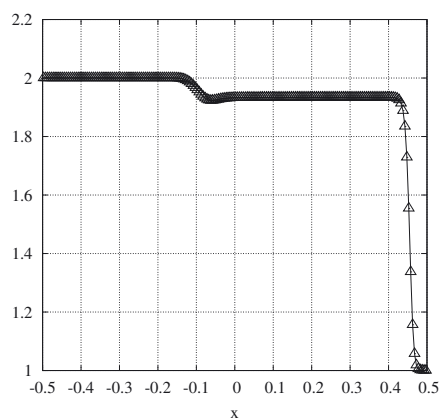

(a) Density

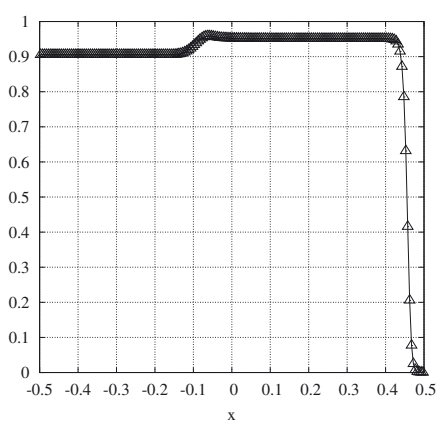

(b) Velocity

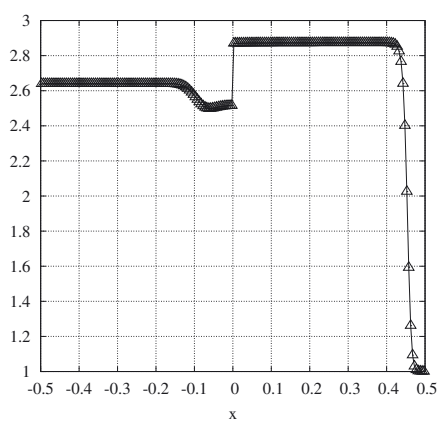

(c) Pressure

FiguRE 7. Non conservative coupling: results for the Relaxation approach.

described in Section 4.2.1 since $\mathcal{M}_{\rho \pi}^{e}(0) \neq 0$ in general. The benefit of this second approach clearly stays in the capture of a perfectly sharp standing wave at the interface. Let us stress that this second method exactly restores by construction the identity $\left(\mathbf{g}_{R}\right)_{0}^{n}=\left(\mathbf{g}_{L}\right)_{0}^{n}$.

\subsection{Non conservative coupling}

In this test case, the value of $\mathcal{M}_{\rho u}$ is chosen so as to enforce the continuity of the traces of the exact Riemann solution in problem 2.1 at $x=0$; namely $\mathbf{u}\left(0^{-}, \mathbf{u}_{L}, \mathbf{u}_{R}\right)=\mathbf{u}\left(0^{+}, \mathbf{u}_{L}, \mathbf{u}_{R}\right)$. It can be seen [20] that the expected value is given by:

$$
\mathcal{M}_{\rho u}^{n}=0.354404, \quad n>0 .
$$

Figures 6 and 7 compare the discrete solutions obtained by the two-Riemann problem approach and the relaxation strategy. The discrete standing waves are quite comparable. Then Figures $8 \mathrm{~b}$ and $9 \mathrm{~b}$ display the component of impulsion of the difference $\left(\mathbf{g}_{R}\right)_{0}^{n}-\left(\mathbf{g}_{L}\right)_{0}^{n}$ and the exact value of $\mathcal{M}_{\rho u}^{n}$ used here. Again by construction such a difference is strictly equal to the prescribed weight at all time steps in the relaxation strategy while it takes few time steps in the two-Riemann problem method.

\section{Conclusion}

We have proposed two well-balanced numerical methods to deal with the non-conservative coupling of two one-dimensional barotropic Euler equations with a Dirac source term to model singular pressure losses. The first method relies on ghost state reconstructions at the coupling interface while the second one is derived thanks to 


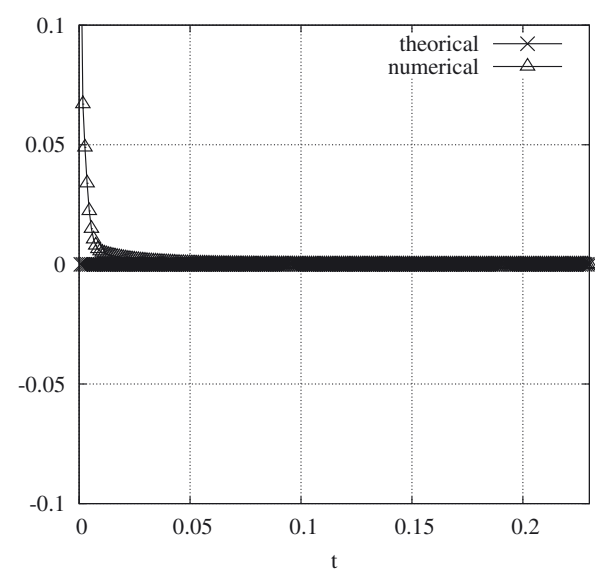

(a) Two Riemann problems: conservative coupling

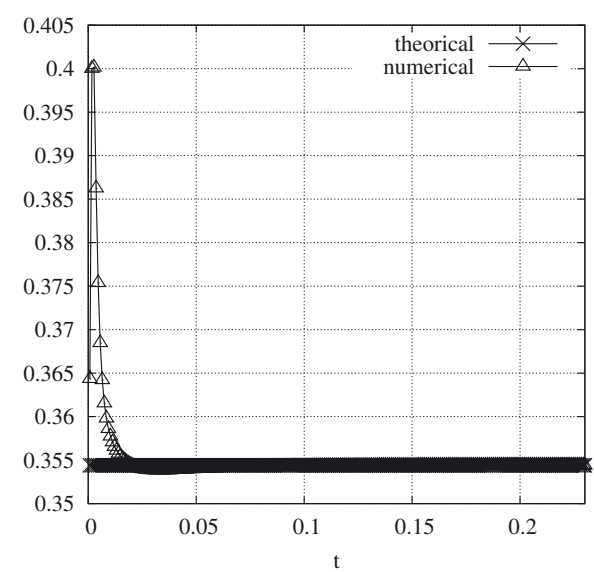

(b) Two Riemann problems: non conservative coupling

FIGURE 8. Comparison between the numerical value of the impulsion flux jump at the interface and the real weight value for the two-Riemann problem approach.

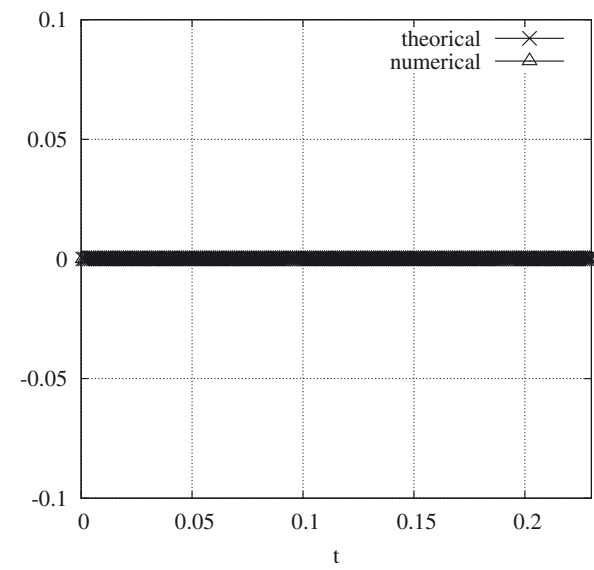

(a) Relaxation approach: conservative coupling

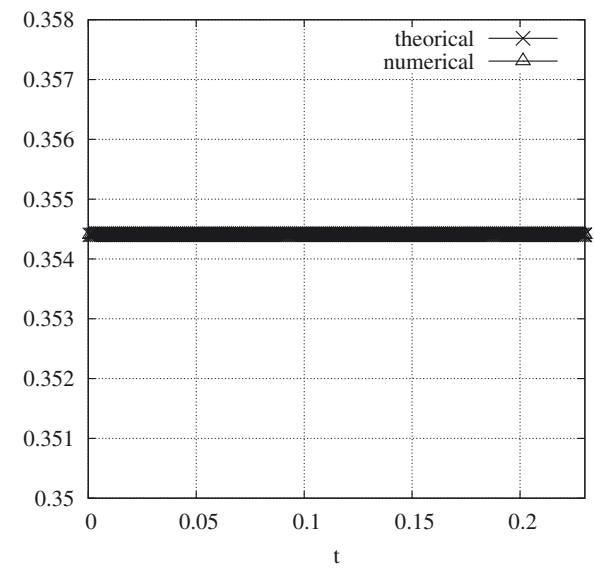

(b) Relaxation approach: non conservative coupling

FiguRE 9. Comparison between the numerical value of the impulsion flux jump at the interface and the real weight value for the relaxation approach.

a suitable extension of a classical pressure relaxation framework. Unlike the first method, the second one always preserves mass conservation and exactly restores the prescribed singular pressure drops for both unsteady and steady solutions. This is the very novelty of the present work. Both methods are first order accurate. Their extension to higher order of approximation together with applications to two-phase flow models will be the matter of a forthcoming study.

Acknowledgements. This work fits in a joint research program between CEA Saclay and Laboratoire Jacques Louis Lions Université Pierre et Marie Curie Paris6, on coupling methods for multiphase flows. This work was partially supported by the NEPTUNE project, funded by CEA, EDF, IRSN and AREVA-NP. 


\section{REFERENCES}

[1] M.S. Adimurthi and G.D.V. Gowda, Optimal entropy solutions for conservation laws with discontinuous flux-functions. J. Hyperbolic Differ. Equ. 2 (2005) 783-837.

[2] D. Amadori, L. Gosse, G. Graziano, Godunov-type approximation for a general resonant balance law with large data. J. Differ. Equ. 198 (2004) 233-274.

[3] A. Ambroso, C. Chalons, F. Coquel, E. Godlewski, F. Lagoutiere, P.-A. Raviart and N. Seguin, The coupling of homogeneous models for two-phase flows. Int. J. Finite Volumes 4 (2007) 1-39.

[4] A. Ambroso, C. Chalons, F. Coquel, E. Godlewski, F. Lagoutiere, P.-A. Raviart and N. Seguin, Coupling of general Lagrangian systems. Math. Comput. 77 (2008) 909-941.

[5] A. Ambroso, J.-M. Hérard and O. Hurisse, A method to couple HEM and HRM two-phase flow models. Comput. Fluids 38 (2009) 738-756.

[6] E. Audusse and B. Perthame, Uniqueness for scalar conservation laws with discontinuous flux via adapted entropies. Proc. Roy. Soc. Edinburgh Sect. A 135 (2005) 253-265.

[7] F. Bachmann and J. Vovelle, Existence and uniqueness of entropy solution of scalar conservation laws with a flux function involving discontinuous coefficients. Commun. Partial Differential Equations 31 (2006) 371-395.

[8] D. Bestion, M. Boucker, P. Boudier, P. Fillion, M. Grandotto, A. Guelfi, J.M. Hérard, E. Hervieu, P. Péturaud, Neptune: a new software platform for advanced nuclear thermal hydraulics. Nuclear Science and Engineering 156 (2007) 281-324.

[9] F. Bouchut, Nonlinear stability of Finite volume methods for hyperbolic conservation laws, and well-balanced schemes for sources. Frontiers in Mathematics, Birkhauser (2004).

[10] B. Boutin, C. Chalons and P.A. Raviart, Existence result for the coupling problem of two scalar conservation laws with Riemann initial data. Math. Models Methods Appl. Sci. 20 (2010) 1859-1898.

[11] B. Boutin, F. Coquel and P.G. LeFloch, Coupling techniques for nonlinear hyperbolic equations. I. Self-similar diffusion for thin interfaces. Proc. Roy. Soc. Edinburgh Sect. A 141 (2011) 921-956.

[12] M. Bucci and P. Fillion, Analysis of the NUPEC PSBT Tests with FLICA-OVAP. Science and Technology of Nuclear Installations. Article ID 2012 (2012) 436142.

[13] R. Bürger and K.H. Karlsen, Conservation laws with discontinuous flux: a short introduction. J. Engrg. Math. 60 (2008) $241-247$.

[14] R. Bürger, K.H. Karlsen and J.D. Towers, An Engquist-Osher-type scheme for conservation laws with discontinuous flux adapted to flux connections. SIAM J. Numer. Anal. 47 (2009) 1684-1712.

[15] C. Chalons, P.-A. Raviart and N. Seguin, The interface coupling of the gas dynamics equations. Quaterly of Applied Mathematics 66 (2008) 659-705.

[16] C.M. Dafermos, Solution of the Riemann problem for a class of hyperbolic systems of conservation laws by the viscosity method. Arch. Rational Mech. Anal. 52 (1973) 1-9.

[17] S. Diehl, On scalar conservation laws with point source and discontinuous flux function. SIAM J. Math. Anal. 26 (1995) $1425-1451$.

[18] S. Diehl, Scalar conservation laws with discontinuous flux function. I. The viscous profile condition, Commun. Math. Phys. 176 (1996) 23-44.

[19] W.H. Hager, Wastewater Hydraulics, Theory and Practice. Springer (2010).

[20] T. Galié, Couplage interfacial de modèles pour la thermoohydraulique des réacteurs, Ph.D. thesis, Université Pierre et Marie Curie Paris 6 (2008).

[21] T. Gimse and N.H. Risebro, Solution of the Cauchy problem for a conservation law with a discontinuous flux function. SIAM J. Math. Anal. 23 (1992) 635-648.

[22] J. Glimm, D. Marchesin and O. McBryan, Numerical method for two phase flow with unstable interface. J. Comput. Phys. 39 (1981) 179-200.

[23] P. Goatin and P.G. LeFloch, The Riemann problem for a class of resonant hyperbolic systems of balance laws. Ann. Inst. H. Poincaré Anal. Non Linéaire 21 (2004) 881-902.

[24] E. Godlewski, K.-C. Le Thanh and P.-A. Raviart, The numerical interface coupling of nonlinear hyperbolic systems of conservation laws. II. The case of systems. M2AN Math. Model. Numer. Anal. 39 (2005) 649-692.

[25] E. Godlewski and P.-A. Raviart, The numerical interface coupling of nonlinear hyperbolic systems of conservation laws. I. The scalar case. Numer. Math. 97 (2004) 81-130.

[26] L. Gosse, A well-balanced scheme using non-conservative products designed for hyperbolic systems of conservation laws with source terms. Math. Models Methods Appl. Sci. 11 (2001) 339-365.

[27] L. Gosse, Localization effects and measure source terms in numerical schemes for balance laws. Math. Comp. 71 (2001) 553-582.

[28] J.M. Greenberg and A.Y. Leroux, A well-balanced scheme for the numerical processing of source terms in hyperbolic equations. SIAM J. Numer. Anal. 33 (1996) 1-16.

[29] E. Isaacson and B.J. Temple, Nonlinear resonance in systems of conservation laws. SIAM J. Appl. Math. 52 (1992) $1260-1278$.

[30] J.M. Greenberg, A.Y.L. Roux, R. Baraille and A. Noussair, Analysis and approximation of conservation laws with source terms. SIAM J. Numer. Anal. 34 (1997) 1980-2007.

[31] J.-M. Hérard, Schemes to couple flows between free and porous medium. Proceedings of AIAA (2005) $2005-4861$.

[32] J.-M. Hérard and O. Hurisse, Coupling two and one-dimensional models through a thin interface. Proceedings of AIAA (2005) $2005-4718$. 
[33] J.-M. Hérard and O. Hurisse, Boundary conditions for the coupling of two-phase flow models. 18th AIAA CFD conference.

[34] I.E. Idel'cik, Memento des pertes de charges. Coefficients de pertes de charges singulières et de pertes de charges par frottement. Collection Direction des Etudes et Recherches d'EDF. Eyrolles [in French] (1986).

[35] S. Jin and Z. Xin, The relaxation schemes for systems of conservation laws in arbitrary space dimensions. Comm. Pure Appl. Math. 48 (1995) 235-276.

[36] V.G. Kourakos, P. Rambaud, S. Chabane and J.M. Buchlin, Modeling of pressure drop in two-phase flow in singular geometries. 6th International Symposium on Multiphase Flow, Heat Mass Transfert and Energy Conservation. Xi'an, China, 11-15 July 2009, Paper No MN-30, 2009.

[37] D.S. Miller (Ed.), Discharge Characteristics: IAHR Hydraulic Structures Design Manuals 8. Balkema: Rotterdam (1994).

[38] S.N. Kruzkov, First order quasilinear equations with several independent variables. Mat. Sb. (N.S.) 81 (1970) $228-255$. 